\title{
Model uncertainty on commodity portfolios, the role of convenience yield
}

\author{
Junhe Chen ${ }^{1} \cdot$ Marcos Escobar-Anel $^{1}$
}

Received: 2 February 2021 / Accepted: 18 June 2021 / Published online: 7 July 2021

(c) The Author(s), under exclusive licence to Springer-Verlag GmbH Germany, part of Springer Nature 2021

\begin{abstract}
This paper investigates the effect of model uncertainty on the performance of commodity-based portfolios. We consider a constant relative risk aversion (CRRA) utility maximizer investor in a complete market, with independent ambiguity-aversion levels for the three factors explaining the term structure of future prices, namely, spot prices, convenience yield (CY) and interest rates (IRs), as proposed in the seminal work of Schwartz (J Finance 52(3): 923-973, 1997). This generic investor is interested in the speculative component of the investment rather than possessing/consuming the physical commodity. We obtain closed-form solutions for optimal investments, optimal perturbations (alternative model) and value functions in line with the robust portfolio setting of Maenhout (Rev Financial Stud 17(4): 951-983, 2004). Our main focus is on the effect of convenience yield's uncertainty on the optimal analysis. We estimate the model by applying a combination of maximum likelihood estimation (MLE) and Kalman Filter (KF) techniques, to two commodities: West Texas Intermediate (WTI) and copper future prices. The analysis demonstrates that uncertainty on the CY factor could be the largest contributor to the under-performance of a commodities portfolio, with wealth equivalent losses (WELs) in the ranges of $33 \%$ to $88 \%$ (WTI), and $7 \%$ to $31 \%$ (copper). Moreover, small variations, of up 25\%, on CY's covariance parameters could lead to a WEL of up to $40 \%$ (WTI, lesser volatility of CY).
\end{abstract}

Keywords Multivariate portfolio choice - Ambiguity aversion ·

Commodity markets · Welfare-equivalent losses

JEL Classification G11 · C61

Marcos Escobar-Anel

marcos.escobar@uwo.ca

Junhe Chen

jche64@uwo.ca

1 Department of Statistical and Actuarial Sciences, Western Science Centre, Western University, 1151 Richmond Street, London, ON, Canada 


\section{Introduction}

A portfolio optimization problem studies optimal asset allocations that maximize investors overall level of wealth. In this paper we focus on investors who want to maximize the expected utility of terminal wealth. Following the modern continuous-time portfolio optimization scheme of Merton (1971), which includes a stock following a geometric Brownian motion (GBM) and a risk-free bank account, considerable works regarding portfolio optimization have been carried out during the past 50 years. New studies have revised many of the underlying assumptions. For instance, the simple GBM model has been extended to incorporate stylized facts such as stochastic volatility (see Kraft 2005), stochastic correlation (Escobar et al. 2016) and mean reverting behaviour (Chiu and Wong 2013). Additional state variables, for example stochastic interest rate (IR) (see Kraft (xxx); Flor and Larsen (2013)) and stochastic convenience yield (CY) (Mellios et al. (2016)), have been included in the analysis with the corresponding need for completeness in the portfolio (see Liu et al. (2005)).

More recently, the notion of certainty in the specification of the parameters has been challenged. With regard to this latest aspect, the seminal work of Anderson et al. (2003) presents the so-called robust optimal control framework, also known as model-misspecification or ambiguity-aversion analysis. Here, an investor has a favorite portfolio allocation derived from a so-called "reference model", but the study also acknowledges that the model may be mis-specified. The investor then studies optimal allocations based on a family of alternative models and settles for a worst-case scenario, namely, the optimal alternative model; this is compatible with the theory of Gilboa and Schmeidler (1989). Maenhout (2004) adapted the robust control framework of Anderson et al. (2003) to a continuous-time stochastic control problem, using the asset setting of Merton (1971), leading to closed-form solutions thanks to the use of Hamilton-Jacobi-Bellman-Issacs (HJBI) equations. After the work of Maenhout (2004), various studies have been completed, providing practitioners with valuable insight into the influence of ambiguity-aversion on portfolio decisions. For example, Liu (2011) considered the ambiguity of the stock return, which followed a Markovswitching process. Furthermore, Flor and Larsen (2013) analyzed the case of one stock and a bond by taking a mean-reverting IR model, and Munk and Rubtsov (2014) considered inflation, stock price and IR to be ambiguous. Finally, Escobar et al. (2015) and Bergen et al. (2018) studied ambiguity aversion in relation to stochastic volatility and stochastic covariance respectively.

Surprisingly, the topics of portfolio optimization and ambiguity aversion has barely been studied for commodity-based investors. The complexity of commodity modeling is well-known, and most literature highlights several factors that are needed to explain the term structure of future prices. For instance, Schwartz (1997) proposed three factors: spot price, short IRs and CY; while Schwartz and Smith (2000) suggested two factors: short-term and long-term dynamics. In the absence of ambiguity, the leading analysis was provided by Mellios et al. (2016), who established the portfolio optimization problem using a spot commodity, a future contract and a bond; the commodity price model is based on Model III of Schwartz (1997). This is a complete market model, where the market price of risk is assumed to be a function of CY and spot prices. To the best of our knowledge there are only two examples of robust analysis 
in commodities. The first is the theoretical work of Cartea et al. (2016), who considered ambiguity-aversion on the diffusive (short-term factor) and jump (long term) components of a model adjusted for seasonal effects and a single stochastic volatility driver. The authors did not study portfolio allocations in this context. The second study, by Chen et al. (2021), involved a log Ornstein-Uhlenbeck for the spot commodity and stochastic IRs using a combination of Models I and II by Schwartz (1997); the focus was on an insurance-type investor with a Cramer-Lundberg surplus. The authors observed that uncertainty in the commodity asset class is more disruptive to portfolio managers than uncertainty in the equity asset class (i.e. commodity managers shall be more attentive to uncertainty than equity managers).

The aforementioned observation highlights the need for further analysis of uncertainty in portfolios of commodities. In particular, a fundamental question is still unanswered: which source of uncertainty, among, CY, IR and spot prices, is more damaging to investors (i.e. to the performance of the portfolio)? We answer the question in our paper. We take the popular Model III of Schwartz (1997) and consider a constant relative risk aversion (CRRA) utility maximizer portfolio manager investing into a spot commodity, a prepaid forward, a bond and a cash account; this ensures a complete market analysis, which has proven to be the most beneficial to investors. We formulate the robust portfolio optimization problem by considering model uncertainty in all three diffusions drivers in the model, namely, spot price, IR and CY, hence attaching a level of ambiguity-aversion to each one of these factors. The results of this study and the novelties are listed next:

- We obtained closed-form expressions for the four key objects in a robust portfolio analysis, namely, optimal investment allocation, optimal alternative model, optimal terminal wealth and value function.

- The three factors model was calibrated to empirical data via a combination of maximum likelihood estimation (MLE) and Kalman filter (KF) techniques. Moreover, we used the West Texas Intermediate (WTI) spot and future prices, as well as the 1-month Treasury Constant Maturity Rate. We also studied a second commodity, copper future prices, to strengthen the results (see Sect. B).

- The empirical analysis established three key insights. First, small changes in CYs' parameters lead to large variations in forward contract allocation; second, small variations in CY covariance parameters could lead to substantial wealth-equivalent losses (WELs); and lastly and more importantly, uncertainty on CY could be the largest contributor to the under-performance of the portfolio.

The paper is structured as follows. Section 2 describes the mathematical model and the robust optimization problem. The analytical solutions are then detailed in Sect. 3. Thereafter, Sect. 4 first explains the estimation of parameters, before exploring optimal investment allocation with regard to the covariance parameters of CY. A WEL analysis is also performed for several key suboptimal cases, namely, parametric misspecification of CY, incompleteness of the market, and more importantly the impact of ignoring the various sources of uncertainty. Section 5 concludes the paper. 


\section{Mathematical settings}

Model III of Schwartz (1997) is presented first in this section. The model considers stochastic spot prices, stochastic IR and stochastic CY, with the latter two following mean-reverting processes. Some results regarding the model-implied term structure of future prices, bond prices and prepaid forwards are described. The robust portfolio optimization problem is then introduced.

\subsection{Three-factor model}

We assume that the stochastic processes describing the financial market are defined on a complete probability space $(\Omega, \mathcal{F}, \mathbb{Q})$ with a right-continuous filtration $\left\{\mathcal{F}_{t}\right\}_{t \in[0, T]}$. Schwartz (1997) presented Model III on the risk-neutral probability measure $\mathbb{Q}$. Let $S(t)$ denote the spot price of a commodity; the model can then be described via three stochastic differential equations (SDEs):

$$
\left\{\begin{array}{l}
\mathrm{d} S(t)=(r(t)-\delta(t)) S(t) \mathrm{d} t+\sigma_{S} S(t) \mathrm{d} W_{1}^{Q}(t) \\
\mathrm{d} \delta(t)=\kappa(\hat{\alpha}-\delta(t)) \mathrm{d} t+\sigma_{\delta} \mathrm{d} W_{2}^{Q}(t) \\
\mathrm{d} r(t)=a(\hat{m}-r(t)) \mathrm{d} t-\sigma_{r} \mathrm{~d} W_{3}^{Q}(t)
\end{array}\right.
$$

with correlations among all Brownian motions,

$$
\begin{aligned}
& \mathrm{d} W_{1}^{Q}(t) \mathrm{d} W_{2}^{Q}(t)=\rho_{S \delta} \mathrm{d} t, \quad \mathrm{~d} W_{2}^{Q}(t) \mathrm{d} W_{3}^{Q}(t)=\rho_{\delta r} \mathrm{~d} t \\
& \mathrm{~d} W_{1}^{Q}(t) \mathrm{d} W_{3}^{Q}(t)=\rho_{S r} \mathrm{~d} t .
\end{aligned}
$$

Here the CY $\delta(t)$ and IR $r(t)$ are mean-reverting processes with reverting rates of $\kappa$ and $a$ and mean values of $\hat{\alpha}$ and $\hat{m}$, respectively. Moreover, CY is the benefit to the investor while holding the commodity. If CY is negative, then it is equivalent to having a cost of carry. Therefore, if an investor holds the commodity directly, then the reduced amount of return on the commodity offsets the benefit; this is similar to the behavior of dividends in a stock.

The investment problem deals with the returns on the real-world probability $\mathbb{P}$. We use Girsanov's change of measure with $\mathrm{d} \boldsymbol{W}^{Q}(t)=\mathrm{d} \boldsymbol{W}(t)+\lambda \mathrm{d} t$ where we set

$$
\lambda=\left[\begin{array}{c}
\lambda_{S} \\
\lambda_{\delta} \\
\lambda_{r}
\end{array}\right], \quad \boldsymbol{W}(t)=\left[\begin{array}{l}
W_{1}(t) \\
W_{2}(t) \\
W_{3}(t)
\end{array}\right] \quad \boldsymbol{W}^{Q}(t)=\left[\begin{array}{c}
W_{1}^{Q}(t) \\
W_{2}^{Q}(t) \\
W_{3}^{Q}(t)
\end{array}\right] .
$$

Hence, $\lambda_{S}, \lambda_{\delta}, \lambda_{r}$ are the market prices of risks of the commodity, CY and IR. Under $\mathbb{P}$, Eq. 1 becomes

$$
\left\{\begin{array}{l}
\mathrm{d} S(t)=\left(r(t)+\lambda_{S} \sigma_{S}-\delta(t)\right) S(t) \mathrm{d} t+\sigma_{S} S(t) \mathrm{d} W_{1}(t) \\
\mathrm{d} \delta(t)=\kappa(\alpha-\delta(t)) \mathrm{d} t+\sigma_{\delta} \mathrm{d} W_{2}(t) \\
\mathrm{d} r(t)=a(m-r(t)) \mathrm{d} t-\sigma_{r} \mathrm{~d} W_{3}(t)
\end{array}\right.
$$


where

$$
\alpha=\hat{\alpha}+\frac{\lambda_{\delta} \sigma_{\delta}}{\kappa}, \quad m=\hat{m}-\frac{\lambda_{r} \sigma_{r}}{a} .
$$

We refer to Eq. 4 as the reference model.

\subsection{Dynamics of assets}

Similar to Mellios et al. (2016), we consider three types of assets in which to invest, in addition to the cash account. These assets are a spot commodity, a prepaid forward and a bond. The dynamics of the last two assets are specified next.

\subsubsection{Dynamics of prepaid forward}

In the real world, commodities are usually traded in the form of futures but not immediately; these are called prepaid forwards. We first need the process of a risk-neutral future price for a maturity $T$, as Schwartz (1997) indicated:

$$
F(S, \delta, r, T)=\mathbb{E}^{Q}[S(T)]=S \exp \left[-\frac{\delta\left(1-\mathrm{e}^{-\kappa T}\right)}{\kappa}+\frac{r\left(1-\mathrm{e}^{-a T}\right)}{a}+C(T)\right],
$$

where $C(T)$ satisfies the formula

$$
\begin{aligned}
C(T)= & \frac{\left(\kappa \hat{\alpha}+\sigma_{S} \sigma_{\delta} \rho_{S \delta}\right)\left(\left(1-\mathrm{e}^{-\kappa T}\right)-\kappa T\right)}{\kappa^{2}} \\
& -\frac{\sigma_{\delta}^{2}\left(4\left(1-\mathrm{e}^{-\kappa T}\right)-\left(1-\mathrm{e}^{-2 \kappa T}\right)-2 \kappa T\right)}{4 \kappa^{3}} \\
& -\frac{\left(a \hat{m}+\sigma_{S} \sigma_{r} \rho_{S r}\right)\left(\left(1-\mathrm{e}^{-a T}\right)-a T\right)}{a^{2}} \\
& -\frac{\sigma_{r}^{2}\left(4\left(1-\mathrm{e}^{-a T}\right)-\left(1-\mathrm{e}^{-2 a T}\right)-2 a T\right)}{4 a^{3}} \\
& +\sigma_{\delta} \sigma_{r} \rho_{\delta r}\left(\frac{\left(1-\mathrm{e}^{-\kappa T}\right)+\left(1-\mathrm{e}^{-a T}\right)-\left(1-\mathrm{e}^{-(a+\kappa) T}\right)}{\kappa a(\kappa+a)}\right. \\
& \left.+\frac{a^{2}\left(1-\mathrm{e}^{-\kappa T}\right)+\kappa^{2}\left(1-\mathrm{e}^{-a T}\right)-\kappa a^{2} T-a \kappa^{2} T}{\kappa^{2} a^{2}(\kappa+a)}\right)
\end{aligned}
$$

Given that the future price is the delivery price at time $T$, we would need to discount this future price to present value to obtain the prepaid forward, mathematically,

$$
P(S, \delta, r, T)=\mathbb{E}^{Q}\left[\exp \left(-\int_{0}^{T} r(s) \mathrm{d} s\right) S(T) \mid \mathcal{F}_{t}\right]
$$


where $P$ denotes the price of a prepaid forward for the commodity. Schwartz (1997) presented the PDE of the prepaid forward as well as its solution:

$$
\begin{aligned}
& \frac{1}{2} \sigma_{S}^{2} S^{2} P_{S S}+\frac{1}{2} \sigma_{\delta}^{2} P_{\delta \delta}+\frac{1}{2} \sigma_{r}^{2} P_{r r}+\sigma_{S} \sigma_{\delta} \rho_{S \delta} S P_{S \delta}+\sigma_{r} \sigma_{\delta} \rho_{\delta r} P_{\delta r}+\sigma_{S} \sigma_{r} \rho_{S \delta} S P_{S r} \\
& \quad+(r-\delta) S P_{S}+\kappa(\hat{\alpha}-\delta) P_{\delta}+a\left(m^{*}-r\right) P_{r}-P_{T}=r P . \\
& P(S, \delta, r, T)=S \exp \left[-\frac{\delta\left(1-\mathrm{e}^{-\kappa T}\right)}{\kappa}+D(T)\right] .
\end{aligned}
$$

where

$$
\begin{aligned}
D(T)= & \frac{\left(\kappa \hat{\alpha}+\sigma_{S} \sigma_{\delta} \rho_{S \delta}\right)\left(\left(1-\mathrm{e}^{-\kappa T}\right)-\kappa T\right)}{\kappa^{2}} \\
& -\frac{\sigma_{\delta}^{2}\left(4\left(1-\mathrm{e}^{-\kappa T}\right)-\left(1-\mathrm{e}^{-2 \kappa T}\right)-2 \kappa T\right)}{4 \kappa^{3}} .
\end{aligned}
$$

Therefore, by setting the maturity to be $T-t$ and denoting $P_{T}(t):=P(S, \delta, r, T-$ $t$ ), the dynamic of this prepaid forward is

$$
\mathrm{d} P_{T}(t)=r(t) P_{T}(t) \mathrm{d} t+\frac{\partial P_{T}(t)}{\partial S} S \sigma_{S} \mathrm{~d} W_{1}^{Q}(t)+\frac{\partial P_{T}}{\partial \delta}(t) \sigma_{\delta} \mathrm{d} W_{2}^{Q}(t) .
$$

Going back to the real-world probability, the dynamic would be

$$
\frac{\mathrm{d} P_{T}(t)}{P_{T}(t)}=\left(r(t)+\lambda_{S} \sigma_{S}-A_{T-t} \lambda_{\delta} \sigma_{\delta}\right) \mathrm{d} t+\sigma_{S} \mathrm{~d} W_{1}(t)-A_{T-t} \sigma_{\delta} \mathrm{d} W_{2}(t) .
$$

where $A_{T-t}=\frac{1-\mathrm{e}^{-\kappa(T-t)}}{\kappa} \geq 0$.

This dynamic indicates that the value of this prepaid forward $P_{T}(t)$ is affected by two Brownian motions: $W_{1}^{Q}(t)$ and $W_{2}^{Q}(t)$. Moreover, the excess returns (i.e. $\left.\lambda_{S} \sigma_{S}-A_{T-t} \lambda_{\delta} \sigma_{\delta}\right)$ on prepaid forwards are also impacted by their maturities. Larger maturities $T-t$ result in smaller returns and smaller volatilities.

\subsubsection{Dynamics of bonds}

We consider investing in a bond due to the presence of a stochastic IR $r(t)$. The price of the bond with a maturity of $T$ under the risk-neutral measure is as follows:

$$
P(t, r(t) ; T):=P(t, r(t))=\exp \left(-I_{T-t} r(t)+J_{T-t}\right)
$$

where

$$
\begin{aligned}
I_{T-t} & =\frac{1-\mathrm{e}^{-\kappa(T-t)}}{\kappa} \\
J_{T-t} & =\left(\bar{r}-\frac{\sigma_{r}^{2}}{2 \kappa^{2}}\right)(I(t ; T)-(T-t))-\frac{\sigma_{r}^{2}}{4 \kappa} I_{T-t}^{2} .
\end{aligned}
$$


For simplicity, we can define the "infinity yield" as $y_{\infty}:=\bar{r}-\frac{\sigma_{r}^{2}}{2 \kappa^{2}}$.

In the risk-neutral measure, the bond price follows a PDE:

$$
P_{t}+P_{r}(\kappa(\bar{r}-r))+\frac{\sigma_{r}^{2}}{2} P_{r r}=r P .
$$

Transforming to a real-world measure using Girsanov's theorem, the joint dynamics for the bond and the IR are

$$
\begin{aligned}
\mathrm{d} r(t) & =\left[\kappa(\bar{r}-r(t))-\lambda_{r} \sigma_{r}\right] \mathrm{d} t-\sigma_{r} \mathrm{~d} W_{1}(t) \\
\mathrm{d} P(t, r(t)) & =\left(r(t)+\lambda_{r} \sigma_{r} I_{T-t}\right) P(t, r(t)) \mathrm{d} t+I_{T-t} \sigma_{r} P(t, r(t)) \mathrm{d} W_{1}(t) .
\end{aligned}
$$

Therefore, for the return on the bond $\lambda_{r} \sigma_{r} I_{T-t}$ is the excess return.

\subsection{Portfolio wealth process}

Now, we consider a portfolio consisting of multiple prepaid forwards with different maturities $T_{1}, \ldots, T_{n}$, a bank account and the bond. Let $\pi_{1}, \pi_{2}, \ldots, \pi_{n}$ and $\pi_{n+1}$ be the amount of wealth invested into the $n$ prepaid forwards and the bond respectively. We write $\nabla[0, T]$ for the set of all admissible strategies; that is, $\mathcal{F}_{t}$-progressively measurable processes with sufficient integrability conditions for the optimization problem to be well defined.

We work with portfolio managers who prefer to invest in the commodity rather than to physically possess or consume it; they use what is called rollover investments. Here, we consider rollover investment of the prepaid forwards and the bond by setting $\tau_{i}=T_{i}-t$ and $\tau$ respectively. In this setting, the dynamic of the portfolio wealth will be

$$
\mathrm{d} X(t)=\left(r(t) X(t)+\boldsymbol{\pi}^{T} \boldsymbol{\sigma} \lambda\right) \mathrm{d} t+\boldsymbol{\pi}^{T} \boldsymbol{\sigma} \mathrm{d} \boldsymbol{W}(t)
$$

where

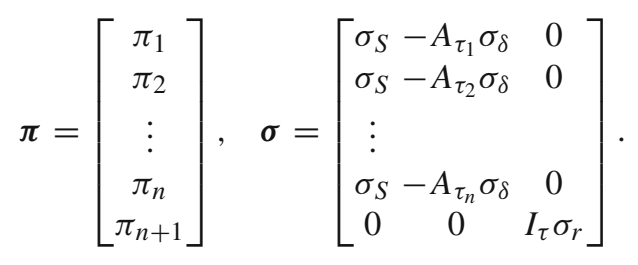

\subsection{The robust portfolio optimization problem}

As per the setting of Maenhout (2004), there is a reference model, Eq. 4, which offers the best representation of the data to the investor. Alternative models also exist that cannot be statistically distinguished from the former model. Girsanov's theorem for correlated Brownian motions allow us to generate alternative models via a perturbation $u$ : 


$$
\frac{\mathrm{d} Q^{u}}{\mathrm{~d} P}=\exp \left(-\int_{0}^{T} \boldsymbol{u}^{T}(t) \mathrm{d} \boldsymbol{W}(t)-\int_{0}^{T} \boldsymbol{u}^{T}(t) \rho^{-1} \boldsymbol{u}(t) \mathrm{d} t\right)
$$

where

$$
\boldsymbol{\rho}=\left[\begin{array}{ccc}
1 & \rho_{S \delta} & \rho_{S r} \\
\rho_{S \delta} & 1 & \rho_{\delta r} \\
\rho_{\delta r} & \rho_{S \delta} & 1
\end{array}\right], \mathrm{d} \boldsymbol{W}(t)=\left[\begin{array}{l}
\mathrm{d} W_{1}(t) \\
\mathrm{d} W_{2}(t) \\
\mathrm{d} W_{3}(t)
\end{array}\right] \text {, and } \boldsymbol{u}(t)=\left[\begin{array}{l}
u_{1}(t) \\
u_{2}(t) \\
u_{3}(t)
\end{array}\right]
$$

We write $\mathcal{U}[0, T]$ for the set of all $\mathcal{F}_{t}$-progressively measurable processes such that the Radon-Nikodým derivative process is well defined. Using the change of measure $P$ to $Q^{u}$, the dynamics become

$$
\left\{\begin{array}{l}
\mathrm{d} S(t)=\left(r(t)-\delta(t)+\lambda_{S} \sigma_{S}-\sigma_{S} u_{1}(t)\right) S(t) \mathrm{d} t+\sigma_{S} S(t) \mathrm{d} W_{1}^{Q^{u}}(t) \\
\mathrm{d} \delta(t)=\left[\kappa(\alpha-\delta(t))-\sigma_{\delta} u_{2}(t)\right] \mathrm{d} t+\sigma_{\delta} \mathrm{d} W_{2}^{Q^{u}}(t) \\
\mathrm{d} r(t)=\left[a(m-r(t))+\sigma_{r} u_{3}(t)\right] \mathrm{d} t-\sigma_{r} \mathrm{~d} W_{3}^{Q^{u}}(t)
\end{array}\right.
$$

Therefore, with the changed drift, the dynamic for the prepaid forward becomes

$$
\begin{aligned}
& \frac{\mathrm{d} P_{T}(t)}{P_{T}(t)}=\left(r(t)+\left(\lambda_{S}-u_{1}(t)\right) \sigma_{S}-A_{T-t}\left(\lambda_{\delta}-u_{2}(t)\right) \sigma_{\delta}\right) \mathrm{d} t \\
& \quad+\sigma_{S} \mathrm{~d} W_{1}^{Q^{u}}(t)-A_{T-t} \sigma_{\delta} \mathrm{d} W_{2}^{Q^{u}}(t) .
\end{aligned}
$$

and the wealth process is

$$
\mathrm{d} X(t)=\left(r(t) X(t)+\boldsymbol{\pi}^{T} \boldsymbol{\sigma}(\boldsymbol{\lambda}-\boldsymbol{u}(t))\right) \mathrm{d} t+\pi^{T} \boldsymbol{\sigma} \mathrm{d} \boldsymbol{W} \boldsymbol{Q}^{u}(t) .
$$

Now, we construct the formal optimization problem. We consider an ambiguous investor with CRRA utility who wants to maximize the expected utility from terminal wealth $X_{T}$. As per Anderson et al. (2003), the value function shall include a penalty term that can be interpreted as the relative entropy for deviating from the reference model. We adopt the most analytically favorable setting of Maenhout (2004):

$$
J(t, x, r)=\sup _{\boldsymbol{\pi}} \inf _{\boldsymbol{u}} \mathbb{E}\left[\frac{J(1-\gamma)}{2} \int_{t}^{T} \boldsymbol{u}^{T}(t) \boldsymbol{\beta}_{\rho}^{-1} \boldsymbol{u}(t) \mathrm{d} t+U\left(X_{T}\right)\right]
$$

where $\rho=\boldsymbol{B} \boldsymbol{B}^{T}$ is a Cholesky decomposition with

$$
\boldsymbol{B}=\left[\begin{array}{ccc}
1 & 0 & 0 \\
\rho_{S \delta} & \sqrt{1-\rho_{S \delta}^{2}} & 0 \\
\rho_{S r} & \frac{\rho_{\delta r}-\rho_{S \delta} \rho_{S r}}{\sqrt{1-\rho_{S \delta}^{2}}} & \frac{\sqrt{1-\rho_{\delta r}^{2}-\rho_{S \delta}^{2}-\rho_{S r}^{2}+2 \rho_{S r} \rho_{\delta r} \rho_{S \delta}}}{\sqrt{1-\rho_{S \delta}^{2}}}
\end{array}\right] .
$$


Here, $\boldsymbol{\beta}=\operatorname{diag}\left(\beta_{1}, \beta_{2}, \beta_{3}\right)$ is the diagonal matrix of parameters representing ambiguity-aversion levels on the underlying processes: IR, CY and the spot commodity. For simplicity we have $\boldsymbol{\beta}_{\rho}:=\boldsymbol{B} \boldsymbol{\beta} \boldsymbol{B}^{T}$ and $U(x)=\frac{x^{1-\gamma}}{1-\gamma}$.

\section{Solution to the robust portfolio problem}

Given the wealth process, we derive the HJBI equation for Eq. 24:

$$
\begin{aligned}
& \sup _{\boldsymbol{\pi}} \inf _{\boldsymbol{u}}\left\{J_{t}+J_{x}\left(r x+\boldsymbol{\pi}^{T} \boldsymbol{\sigma}(\boldsymbol{\lambda}-\boldsymbol{u})\right)+\frac{1}{2} J_{x x} \boldsymbol{\pi}^{T} \boldsymbol{\sigma} \boldsymbol{\rho} \boldsymbol{\sigma}^{T} \boldsymbol{\pi}\right. \\
& +\left[a(m-r)+\sigma_{r} u_{r}\right] J_{r}+\frac{1}{2} J_{r r} \sigma_{r}^{2} \\
& \left.-J_{x r} \boldsymbol{\pi}^{T} \boldsymbol{\sigma} \boldsymbol{\rho}_{r} \sigma_{r}+\frac{J(1-\gamma)}{2} \boldsymbol{u}^{T} \boldsymbol{\beta}_{\boldsymbol{\rho}}^{-1} \boldsymbol{u}\right\}=0,
\end{aligned}
$$

where we set $\rho_{r}=\mathrm{d} \boldsymbol{W}(t) \mathrm{d} W_{3}(t)=\left[\rho_{S r}, \rho_{\delta r}, 1\right]^{T} \mathrm{~d} t .27$ gives the solution to Eq. 26, assuming an exponential affine form of the value function.

Proposition 1 The solution to Eq. 26 is $J(t, x, r)=\frac{x^{1-\gamma}}{1-\gamma} \exp \left(A_{0}(t)+A_{1}(t) r\right)$, where

$$
\begin{aligned}
A_{1}(t)= & \frac{1-\gamma}{a}\left(1-\mathrm{e}^{-a(T-t)}\right) \\
A_{0}(t)= & \frac{1-\gamma}{2} \lambda^{T}\left(\gamma \rho+\boldsymbol{\beta}_{\rho}\right)^{-1} \lambda(T-t) \\
+ & \left(a m+\lambda^{T}\left(\gamma \rho+\boldsymbol{\beta}_{\rho}\right)^{-1}\left(-\boldsymbol{\rho}_{r}(1-\gamma)+\boldsymbol{\beta}_{\rho} \boldsymbol{e}_{3}\right) \sigma_{r}\right) \\
& \frac{1-\gamma}{a}\left[T-t-\frac{1}{a}\left(1-\mathrm{e}^{-a(T-t)}\right)\right] \\
+ & \frac{\sigma_{r}^{2}}{2}\left(1-\frac{\boldsymbol{e}_{3}^{T} \boldsymbol{\beta}_{\rho} \boldsymbol{e}_{3}}{1-\gamma}+\frac{1}{1-\gamma}\left(-\boldsymbol{\rho}_{r}(1-\gamma)+\boldsymbol{\beta}_{\rho} \boldsymbol{e}_{3}\right)^{T}\left(\gamma \rho+\boldsymbol{\beta}_{\rho}\right)^{-1}\right. \\
\times & \frac{(1-\gamma)^{2}}{a^{2}}\left[T-t-\frac{2}{a}\left(1-\mathrm{e}^{-a(T-t)}\right)+\frac{1}{2 a}\left(1-\mathrm{e}^{-2 a(T-t)}\right)\right] .
\end{aligned}
$$

Proof See Appendix A.1.

We discuss the completeness of market in 2. This result demonstrates that a complete market requires at least two prepaid forwards (one could be the spot price) and a bond to control for the sources of risk in the model.

Proposition 2 Let $J_{\left.\pi\right|_{m, s}}$ denote the value function when investing in $m$ prepaid forwards, and $s \in\{0,1\}$ indicates the existence of bonds ( $s=1$ if the bond exists, $s=0$ otherwise); then, we have 
$-J_{\left.\pi\right|_{n>2, s}}=J_{\left.\pi\right|_{n=2, s}} \geq\left. J_{\pi}\right|_{n=1, s}$,

$-\left.J_{\pi}\right|_{m, 1} \geq\left. J_{\pi}\right|_{m, 0}$

Proof Proving that $J_{\left.\pi\right|_{n>2, s}} \geq J_{\left.\pi\right|_{n>2, s}}$ is equivalent to proving it in a semi-definite sense. For $n>2$, we can see that the supremum in 49 can be transformed into:

$$
\begin{aligned}
& \sup _{\boldsymbol{\sigma}^{T} \boldsymbol{\pi}}\left\{J_{t}+J_{x} r x+\boldsymbol{\pi}^{T} \boldsymbol{\sigma}\left(J_{x} \boldsymbol{\lambda}-J_{x r} \boldsymbol{\rho}_{r} \sigma_{r}+J_{x} J_{r} \frac{\boldsymbol{\beta}_{\rho} \boldsymbol{e}_{3} \sigma_{r}}{J(1-\gamma)}\right)\right. \\
& +a(m-r) J_{r}+\frac{1}{2} J_{r r} \sigma_{r}^{2} \\
& \left.\quad-\frac{J_{r}^{2} \boldsymbol{e}_{3}^{T} \boldsymbol{\beta}_{\rho} \boldsymbol{e}_{3}}{2 J(1-\gamma)} \sigma_{r}^{2}+\frac{1}{2} \boldsymbol{\pi}^{T} \boldsymbol{\sigma}\left(J_{x x} \boldsymbol{\rho}-\frac{J_{x}^{2}}{J(1-\gamma)} \boldsymbol{\beta}_{\rho}\right) \boldsymbol{\sigma}^{T} \boldsymbol{\pi}\right\}=0 .
\end{aligned}
$$

In our setting, the matrix $J_{x x} \rho-\frac{J_{x}^{2}}{J(1-\gamma)} \boldsymbol{\beta}_{\rho}$ is assumed to have a full rank of 3 . Therefore, to achieve the supremum, one must have $\operatorname{span}\left(\sigma^{T} \pi\right)=\mathbb{R}^{3}$, which can be ensured if $n \geq 2$ with the form of $\sigma$ in 18 .

However, if $n \leq 1$ or $s=0$, then $\operatorname{span}\left(\sigma^{T} \pi\right)$ is a subspace of $\mathbb{R}^{3}$. In this case, the optimal value will not be achieved except when the optimal point $\sigma^{T} \pi^{*}(t)$ is in the subspace, which would not be possible.

This means that, in our setting, the utility of investing into two prepaid forward is higher than investing in one prepaid forward. Moreover, investing in three or more prepaid forwards would not generate a higher utility. Lastly, including a bond investment also leads to a higher utility.

If we have a complete market with the least number of prepaid forwards, the matrix $\sigma$ is an invertible $3 \times 3$ matrix. Then, the optimal investment allocation becomes

$$
\begin{aligned}
\boldsymbol{\pi}^{*}(t)= & -\left(\boldsymbol{\sigma}^{T}\right)^{-1}\left(J_{x x} \boldsymbol{\rho}-\frac{J_{x}^{2}}{J(1-\gamma)} \boldsymbol{\beta}_{\rho}\right)^{-1}\left(J_{x} \lambda-J_{x r} \boldsymbol{\rho}_{r} \sigma_{r}+J_{x} J_{r} \frac{\boldsymbol{\beta}_{\rho} \boldsymbol{e}_{3} \sigma_{r}}{J(1-\gamma)}\right) \\
= & x\left[\sigma\left(\gamma \rho+\boldsymbol{\beta}_{\rho}\right) \sigma^{T}\right]^{-1} \sigma \lambda \\
& +x\left[\sigma\left(\gamma \rho+\boldsymbol{\beta}_{\rho}\right) \sigma^{T}\right]^{-1} \sigma A_{1}(t)\left(-\boldsymbol{\rho}_{r}+\frac{\boldsymbol{\beta}_{\rho} \boldsymbol{e}_{3}}{1-\gamma}\right) \sigma_{r} .
\end{aligned}
$$

The expression of the optimal allocation has two components: a Merton's type, myopic term, which is driven by all three market prices of risk $\lambda$ (i.e. spot price, CY and IR) and a time-dependent term driven by the stochastic nature of the IRs.

In addition, the expression shows that the variability of the proportion of wealth allocated to risky assets over time (i.e. $\frac{\pi}{x}$ ) should be small, given that $\sigma_{r}$, the volatility of IR is sufficiently small. 
Theorem 1 Minkovski's theorem holds for change of measure $\boldsymbol{u}^{*}(t)$, given a well defined $\left|A_{1}(t)\right|<\infty$ for $0 \leq t \leq T$. Hence $\pi^{*}$ is the optimal strategy in the worstcase scenario provided that the change of drift term, $\boldsymbol{u}^{*}(t)$ is a pure function of $t$.

Proof See Appendix A.2.

\subsection{Wealth-equivalent loss analysis}

In this section we study WELs implied by a variety of important suboptimal allocations of the form $\boldsymbol{\pi}_{s}(t)=x \boldsymbol{h}(t)$, where $\boldsymbol{h}(t)$ is a deterministic function.

The objective function associated with the suboptimal strategy $\pi_{s}(t)$ can be defined as follows:

$$
J^{\boldsymbol{\pi}_{s}}(t, x, r)=\inf _{\boldsymbol{u}} \mathbb{E}\left[\frac{J(1-\gamma)}{2} \int_{t}^{T} \boldsymbol{u}^{T}(t) \boldsymbol{\beta}_{\rho}^{-1} \boldsymbol{u}(t) \mathrm{d} t+U\left(X_{T}\right)\right]
$$

The HJB equation for Eq. 30 is

$$
\begin{aligned}
& \inf _{\boldsymbol{u}}\left\{J_{t}+J_{x}\left(r x+\boldsymbol{\pi}_{s}^{T}(t) \boldsymbol{\sigma}(\boldsymbol{\lambda}-\boldsymbol{u})\right)+\frac{1}{2} J_{x x} \boldsymbol{\pi}_{s}^{T}(t) \boldsymbol{\sigma} \rho \boldsymbol{\sigma}^{T} \boldsymbol{\pi}_{s}(t)\right. \\
& \quad+\left[a(m-r)+\sigma_{r} u_{r}\right] J_{r} \\
& \left.\quad+\frac{1}{2} J_{r r} \sigma_{r}^{2}-J_{x r} \boldsymbol{\pi}_{s}^{T} \boldsymbol{\sigma} \boldsymbol{\rho}_{r} \sigma_{r}+\frac{J(1-\gamma)}{2} \boldsymbol{u}^{T} \boldsymbol{\beta}_{\boldsymbol{\rho}}^{-1} \boldsymbol{u}\right\}=0 .
\end{aligned}
$$

The solution to Eq. 31 is given in 3 .

Proposition 3 The optimal solution to Eq. 31 is $J^{\pi_{s}}(t, x, r)=\frac{x^{1-\gamma}}{1-\gamma} \exp \left(A_{0}^{s}(t)+\right.$ $\left.r A_{1}^{s}(t)\right)$, where

$$
\begin{aligned}
A_{1}^{s}(t)= & \frac{1-\gamma}{a}\left(1-\mathrm{e}^{-a(T-t)}\right) \\
A_{0}^{s}(t)= & \int_{t}^{T}-\frac{(1-\gamma)}{2} \boldsymbol{h}^{T}(s) \boldsymbol{\sigma}\left(\gamma \boldsymbol{\rho}+\boldsymbol{\beta}_{\boldsymbol{\rho}}\right) \boldsymbol{\sigma}^{T} \boldsymbol{h}(s)+\frac{1}{2} A_{1}^{2}(s) \sigma_{r}^{2}\left(1-\frac{\boldsymbol{e}_{3}^{T} \boldsymbol{\beta}_{\rho} \boldsymbol{e}_{3}}{1-\gamma}\right) \\
& \left.+a m A_{1}(s)+\boldsymbol{h}^{T}(s) \boldsymbol{\sigma}\left((1-\gamma) \lambda+A_{1}(s)\left(-(1-\gamma) \boldsymbol{\rho}_{r}+\boldsymbol{\beta}_{\rho} \boldsymbol{e}_{3}\right) \sigma_{r}\right)\right] \mathrm{d} s
\end{aligned}
$$

Proof See Appendix A.3.

Now, we are ready to define the wealth-equivalent "utility" loss (WEL), which is the $L$ solution to the equation $J^{\pi_{s}}(t, x, r)=J\left(t, x\left(1-L^{\pi_{s}}\right), r\right)$, this is:

$$
L^{\pi_{s}}=1-\exp \left(\frac{A_{0}^{s}(t)-A_{0}(t)}{1-\gamma}\right),
$$

where one should note that $A_{1}(t)=A_{1}^{s}(t)$. We consider two general families of suboptimal strategies and therefore two sources of WEL. 


\section{Misspecification of parameters}

The most important suboptimal strategies are the result of selecting embedded models; this can hence be seen as wrong choice of parameter values. We denote the set of misspecified parameters with a hat (e.g. $\hat{\sigma})$. Then the suboptimal investment allocation would be:

$$
\boldsymbol{h}(t)=\left[\hat{\boldsymbol{\sigma}}\left(\gamma \hat{\boldsymbol{\rho}}+\hat{\boldsymbol{\beta}}_{\hat{\boldsymbol{\rho}}}\right) \hat{\boldsymbol{\sigma}}^{T}\right]^{-1} \hat{\boldsymbol{\sigma}}\left(\hat{\boldsymbol{\lambda}}+A_{1}(t)\left(-\hat{\boldsymbol{\rho}}_{r}+\frac{\hat{\boldsymbol{\beta}}_{\hat{\boldsymbol{\rho}}} \boldsymbol{e}_{3}}{1-\gamma}\right) \hat{\sigma}_{r}\right) .
$$

Plugging this investment into Eq. 30 returns a closed-form solution for $J^{\pi_{s}}$, which is not listed here for the sake of simplicity. In particular, this allows us to study the impact of misspecification on the single most difficult parameter to estimate from financial time series, namely, the market price of risk (i.e. $\lambda$ ) for an investor who ignores ambiguity aversion on any of the factors (e.g. $\hat{\beta}_{i}=0$ for some $i$ ). The next proposition provides some insight into the implied WEL.

Proposition 4 Keeping all parameters constants, the WEL from assuming the alternative ambiguity matrix $\hat{\boldsymbol{\beta}}$ is a quadratic function of $\lambda$.

Proof See Appendix A.4.

We can therefore study the impact of ignoring the ambiguity-aversion on a particular factor (e.g. first factor) by setting $\hat{\boldsymbol{\beta}}=\operatorname{diag}\left(0, \beta_{2}, \beta_{3}\right)$, in terms of the factor's market price of risk $\left(\lambda_{1}\right)$. As a result, the matrix affecting $\lambda$ is

$$
(\gamma \boldsymbol{I}+\boldsymbol{\beta})^{\frac{1}{2}}(\gamma \boldsymbol{I}+\hat{\boldsymbol{\beta}})^{-1}-(\gamma \boldsymbol{I}+\boldsymbol{\beta})^{-\frac{1}{2}}=\left[\begin{array}{ll}
\frac{\beta_{1}}{\gamma \sqrt{\gamma+\beta_{1}}} & \\
& 0 \\
& 0
\end{array}\right] .
$$

Therefore, the matrix form $\boldsymbol{B}$ in Eq. 25 gives us

$$
\left((\gamma \boldsymbol{I}+\boldsymbol{\beta})^{\frac{1}{2}}(\gamma \boldsymbol{I}+\hat{\boldsymbol{\beta}})^{-1}-(\gamma \boldsymbol{I}+\boldsymbol{\beta})^{-\frac{1}{2}}\right) \boldsymbol{B}^{-1} \boldsymbol{\lambda}
$$

which, together with Eq. 64, means that WEL depends only on $\lambda_{1}$ in a quadratic form.

\section{Incomplete markets}

An incomplete market means a constraint on allocation, in particular $\pi_{i}=0$ for some $i \in\{1,2,3\}$ in Eq. 26 .

$$
\begin{aligned}
& \sup _{\boldsymbol{\pi} \mid \pi_{i}=0} \inf _{\boldsymbol{u}}\left\{J_{t}+J_{x}\left(r x+\boldsymbol{\pi}^{T} \boldsymbol{\sigma}(\boldsymbol{\lambda}-\boldsymbol{u})\right)+\frac{1}{2} J_{x x} \boldsymbol{\pi}^{T} \boldsymbol{\sigma} \boldsymbol{\rho} \boldsymbol{\sigma}^{T} \boldsymbol{\pi}\right. \\
& +\left[a(m-r)+\sigma_{r} u_{3}\right] J_{r}+\frac{1}{2} J_{r r} \sigma_{r}^{2} \\
& \left.\quad-J_{x r} \boldsymbol{\pi}^{T} \boldsymbol{\sigma} \boldsymbol{\rho}_{r} \sigma_{r}+\frac{J(1-\gamma)}{2} \boldsymbol{u}^{T} \boldsymbol{\beta}_{\boldsymbol{\rho}}^{-1} \boldsymbol{u}\right\}=0
\end{aligned}
$$


In this case, the optimal solution to such constrained problem is required, which is presented in the corollary next.

First, let us use the notation " $\mid-1,(i, j) \in\left\{(i, j): \pi_{i}, \pi_{j} \neq 0\right\}$ " for the inverse of the matrix whose rows and columns are constrained so that the corresponding investment allocation is not 0 , while keeping other elements to 0 . For example,

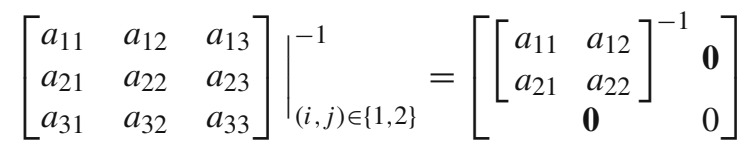

Corollary 1 The optimal investment allocation using Eq. 37 is

$$
\boldsymbol{\pi}^{s}(t)=x \boldsymbol{P} \boldsymbol{\sigma}\left(\lambda+A_{1}(t)\left(-\boldsymbol{\rho}_{r}+\frac{\boldsymbol{\beta}_{\rho} \boldsymbol{e}_{3}}{1-\gamma}\right) \sigma_{r}\right) .
$$

where

$$
\boldsymbol{P}=\left.\left[\boldsymbol{\sigma}\left(\gamma \rho+\boldsymbol{\beta}_{\rho}\right) \boldsymbol{\sigma}^{T}\right]\right|_{(i, j), \pi_{i}, \pi_{j} \neq 0} ^{-1} .
$$

Proof See Appendix A.5.

\section{Empirical analysis}

The data we used to estimate the parameters related to the commodity and CY are spot prices from WTI quotes ${ }^{1}$. We also utilized NYMEX future prices of 1- to 4-month horizons $^{2}$, which are available from the U.S. Energy Information Administration. For IR data, we used the 1-month Treasury Constant Maturity Rate $^{3}$ available from the Federal Reserve Bank of St. Louis. We fit the data between Aug 2001 and Jan 2020, which avoided the shock due to the Covid-19 pandemic.

Similarly to the setting of Mellios et al. (2016), we invested in a spot commodity $(\tau=0)$ and a prepaid forward with a maturity of one quarter $\left(\tau_{2}=1 / 4\right)$. The parameters are estimated in Sect. 4.1 and summarized in Table 1. In particular, the parameters of ambiguity-aversion and risk aversion come from Flor and Larsen (2013), where we assumed a larger range of ambiguity aversion for CY due to its unobservability (hidden Markov process) and therefore the extra difficulty for an investor to trust the reference parameters (higher uncertainty). ${ }^{4}$ Section 4.2 presents the optimal strategies and the WELs from suboptimal strategies.

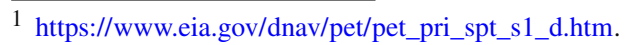

2 https://www.eia.gov/dnav/pet/pet_pri_fut_s1_d.htm.

3 https://fred.stlouisfed.org/series/DGS1MO.

4 See Escobar et al. (2015) for a similar situation of unobservable processes, and a detection-errorprobability analysis concluding aa feasible large range for ambiguity-aversion levels.
} 
Table 1 Parameters from calibration

\begin{tabular}{llll}
\hline Parameters & & & \\
\hline Convenience yield & Mean-reverting rate & $\kappa$ & 4.3799 \\
& Risk-neutral measure & $\hat{\alpha}$ & $-11.94 \%$ \\
& Real-world measure & $\alpha$ & $-3.76 \%$ \\
& Market price of risk & $\lambda_{\delta}$ & 0.9344 \\
Volatility & $\sigma_{\delta}$ & $38.33 \%$ \\
Interest rate & Mean-reverting rate & $a$ & 0.2131 \\
& Real-world measure interest & $m$ & $0.77 \%$ \\
Commodities & Market price of risk & $\lambda_{r}$ & 0.6731 \\
& Volatility & $\sigma_{r}$ & $0.80 \%$ \\
Market price of risk & $\lambda_{S}$ & 0.1322 \\
Correlation & Volatility & $\sigma_{S}$ & $29.86 \%$ \\
& Maturity 1 & $\tau_{1}$ & 0 \\
Maturity 2 & $\tau_{2}$ & $1 / 12$ \\
Objective function & Commodity and CY & $\rho_{S \delta}$ & $49.65 \%$ \\
& Commodity and IR & $\rho_{S r}$ & $3.51 \%$ \\
& IR and CY & $\rho_{r}$ & $-1.04 \%$ \\
& Time horizon & $T$ & 10 \\
& Relative risk aversion & $\gamma$ & 4 \\
& Ambiguity parameter 1 & $\beta_{1}$ & 3 \\
& Ambiguity parameter 2 & $\beta_{2}$ & $3,6,9$ \\
& Ambiguity parameter 3 & $\beta_{3}$ & 3 \\
\hline
\end{tabular}

\subsection{Estimation of parameters}

Referring to Schwartz (1997), the parameters in a real-world measure can be estimated through discretizing 4 . We assume that the time variable $t$ is partitioned into $0=t_{0}<$ $t_{1}<\cdots<t_{N}=T$, where time difference $\Delta t=\frac{T}{N}$.

Define $X_{i}=\ln S\left(t_{i}\right), \delta_{i}=\delta\left(t_{i}\right), r_{i}=r\left(t_{i}\right)$ and $\Delta W_{j}\left(t_{i}\right)=W_{j}\left(t_{i+1}\right)-W_{j}\left(t_{i}\right) \sim$ $\mathrm{N}(0, \Delta t)$. The discretized process is as follows:

$$
\begin{aligned}
X_{i+1}-X_{i} & =\left(r_{i}+\lambda_{S} \sigma_{S}-\delta_{i}-\frac{1}{2} \sigma_{S}^{2}\right) \Delta t+\sigma_{S} \Delta W_{1}\left(t_{i}\right) \\
\delta_{i+1}-\delta_{i} & =\kappa\left(\alpha-\delta_{i}\right) \Delta t+\sigma_{\delta} \Delta W_{2}\left(t_{i}\right) \\
r_{i+1}-r_{i} & =a\left(m-r_{i}\right) \Delta t-\sigma_{r} \Delta W_{3}\left(t_{i}\right)
\end{aligned}
$$

which can help us to obtain the estimates of all above-mentioned parameters and the correlation between the Brownian motions $W_{1}(t), W_{2}(t), W_{3}(t)$.

For the mean-reverting level parameters $\hat{m}$ and $\hat{\alpha}$, we use the expression of $\log$ commodity future price based on Eq. 6 


$$
\ln F_{i, j}=\ln S_{i}-\frac{\delta_{i}\left(1-\mathrm{e}^{-\kappa T}\right)}{\kappa}+\frac{r_{i}\left(1-\mathrm{e}^{-a T}\right)}{a}+C\left(\tau_{j}\right)
$$

where $F_{i, j}:=F\left(S\left(t_{i}\right), \delta\left(t_{i}\right), r\left(t_{i}\right), T_{j}\right)$, and $t_{i}, \tau_{j}$ are current times and the time to maturities respectively. Furthermore, $\hat{m}$ and $\hat{\alpha}$ can be estimated from the formula of $C(T)$ (i.e. Eq. 7).

Given the relationship between the log future price in Eq. 42 and the stochastic processes in Eq. 41, we combine the MLE of parameters with a KF. We create a system of a state-space model with regard to log future prices and CY, as indicated below. Given the data of future prices, spot prices and IR, we define the known term

$$
G_{i, j}:=\ln F_{i, j}-\ln S_{i}-\frac{r_{i}\left(1-\mathrm{e}^{-a T_{j}}\right)}{a}
$$

where $i, j$ represent the time and the time to maturity, respectively. Using the notation of $G_{i, j}$, our state-space model is changed into

$$
\begin{aligned}
\delta_{i+1} & =\kappa \alpha \Delta t+(1-\kappa \Delta t) \delta_{i}+\sigma_{\delta} \Delta W_{2}\left(t_{i}\right) \\
\boldsymbol{G}_{i} & =\boldsymbol{A}+\boldsymbol{K} \delta_{i}+\boldsymbol{v}_{i} .
\end{aligned}
$$

where $w_{i}=\sigma_{\delta} \Delta W_{2}\left(t_{i}\right) \sim N\left(0, \sigma_{\delta}^{2} \Delta t\right), G_{i, j}$ is the given measurement, $\delta_{i}$ is the state model to be estimated, and

$$
\boldsymbol{G}_{i}=\left[\begin{array}{l}
G_{i, 1} \\
G_{i, 2} \\
G_{i, 3} \\
G_{i, 4}
\end{array}\right], \quad \boldsymbol{A}=\left[\begin{array}{l}
C\left(\tau_{1}\right) \\
C\left(\tau_{2}\right) \\
C\left(\tau_{3}\right) \\
C\left(\tau_{4}\right)
\end{array}\right], \quad \boldsymbol{K}=\left[\begin{array}{c}
-\frac{\left(1-\mathrm{e}^{-\kappa T_{1}}\right)}{\kappa} \\
-\frac{\left(1-\mathrm{e}^{-\kappa T_{2}}\right)}{\kappa} \\
-\frac{\left(1-\mathrm{e}^{-\kappa T_{3}}\right)}{\kappa} \\
-\frac{\left(1-\mathrm{e}^{-\kappa T_{4}}\right)}{\kappa}
\end{array}\right], \quad \boldsymbol{v}_{i}=\left[\begin{array}{c}
v_{i, 1} \\
v_{i, 2} \\
v_{i, 3} \\
v_{i, 4}
\end{array}\right] \sim N(0, R)
$$

where we set $R=\operatorname{diag}\left(\sigma_{1}, \sigma_{2}, \sigma_{3}, \sigma_{4}\right)$.

Due to the absence of sufficient time to maturities, we cannot directly estimate all coefficients in $C(T)$ from Eq. 7. Rather, we approximate the term $C(T)$ via Taylor expansion on $\mathrm{e}^{x}$,

$$
\begin{aligned}
& \frac{\left(1-\mathrm{e}^{-\kappa T}\right)-\kappa T}{\kappa^{2}}=\frac{1-\left(1-\kappa T+1 / 2 \kappa^{2} T^{2}+O\left(T^{3}\right)\right)-\kappa T}{\kappa^{2}} \\
& =\frac{1}{2} T^{2}+O\left(T^{3}\right) \\
& \frac{4\left(1-\mathrm{e}^{-\kappa T}\right)-\left(1-\mathrm{e}^{-2 \kappa T}\right)-2 \kappa T}{4 \kappa^{3}}=\frac{4\left(\kappa T-1 / 2 \kappa^{2} T^{2}+O\left(T^{3}\right)\right)}{4 \kappa^{3}} \\
& \quad-\frac{\left(2 \kappa T-1 / 2(2 \kappa T)^{2}+O\left(T^{3}\right)\right)-2 \kappa T}{4 \kappa^{3}}
\end{aligned}
$$




$$
\begin{aligned}
= & O\left(T^{3}\right) \frac{\left(1-\mathrm{e}^{-\kappa T}\right)+\left(1-\mathrm{e}^{-a T}\right)-\left(1-\mathrm{e}^{-(a+\kappa) T}\right)}{\kappa a(\kappa+a)} \\
& +\frac{a^{2}\left(1-\mathrm{e}^{-\kappa T}\right)+\kappa^{2}\left(1-\mathrm{e}^{-a T}\right)-\kappa a^{2} T-a \kappa^{2} T}{\kappa^{2} a^{2}(\kappa+a)} \\
= & O\left(T^{3}\right)
\end{aligned}
$$

By omitting the term $O\left(T^{3}\right)$, the approximation of $C(T)$ is

$$
C(T) \approx\left[\left(\kappa \hat{\alpha}+\sigma_{S} \sigma_{\delta} \rho_{S \delta}\right)-\left(a \hat{m}+\sigma_{S} \sigma_{r} \rho_{S r}\right)\right] \frac{T^{2}}{2}:=b T^{2},
$$

and the parameters to be estimated hence become $b$, and $A=b\left[\tau_{1}^{2}, \tau_{2}^{2}, \tau_{3}^{2}, \tau_{4}^{2}\right]^{T}$.

\subsection{Optimal strategy and suboptimal analysis}

In this section, we first demonstrate the impact of CY parameters on the optimal strategy. Then we study WEL in three different context. First, we analyze WEL for a misspecification of covariance-related parameters in Sect. 4.2.2. This is a type of misspecification not accounted for by ambiguity-aversion in the setting of Maenhout (2004). It addresses the impact on wealth of using a wrong value for a parameter, any strategy produced with the wrong value (e.g. bad estimate) of a parameter would be suboptimal compared to the strategy produced by the true value of the parameter. The section shed light on how serious estimation inaccuracy could be on the WEL.

Section 4.2.3 addresses WEL in a second context, this is as a consequence of an investor disregarding its own true level of ambiguity-aversion. This means the investor acts as if she has perfect knowledge of the true distribution of the underlying factors (stock, interest rate and $\mathrm{CY}$ ), i.e. as if $\beta=0$. Such behaviour would be suboptimal if there is clear evidence, e.g. due to lack of data or expertise, that the investor do not know with total confidence those distributions, i.e. $\beta>0$. Another common reason why investors may choose the $\beta=0$ solution, even when acknowledging aversion to ambiguity, is due to the lack of a mathematical framework and closed-form solutions to implement their intentions (e.g. solely risk-averse solutions are well known); this paper fill this gap for the first time on commodity investors.

It is also important to notice that the ambiguity-averse solution $(\beta>0)$ comes from a worst-case analysis axiomatized in Gilboa and Schmeidler (1989). It is common to think that investors would be better off using a non-ambiguous solution as this would avoid the apparent penalty embedded in taking a worst-case solution. To see the pitfall in the reasoning here, one can use the insight from Sect. 4.2.2; which clearly demonstrates that using the wrong value of the parameter (acting as if it were correct) could lead to huge WEL consequences. The robust analysis theory provides a sound and optimal approach to handle such lack of knowledge.

Lastly, Sect. 4.2.4 focuses on a third context of WEL. Here, we study the implications of investors acting as if the market were incomplete, i.e. not hedging all sources of randomness impacting prices and therefore using the incomplete market solution. Such solution would be suboptimal in the context of solutions hedging all risk (a.k.a 
Fractional Investment
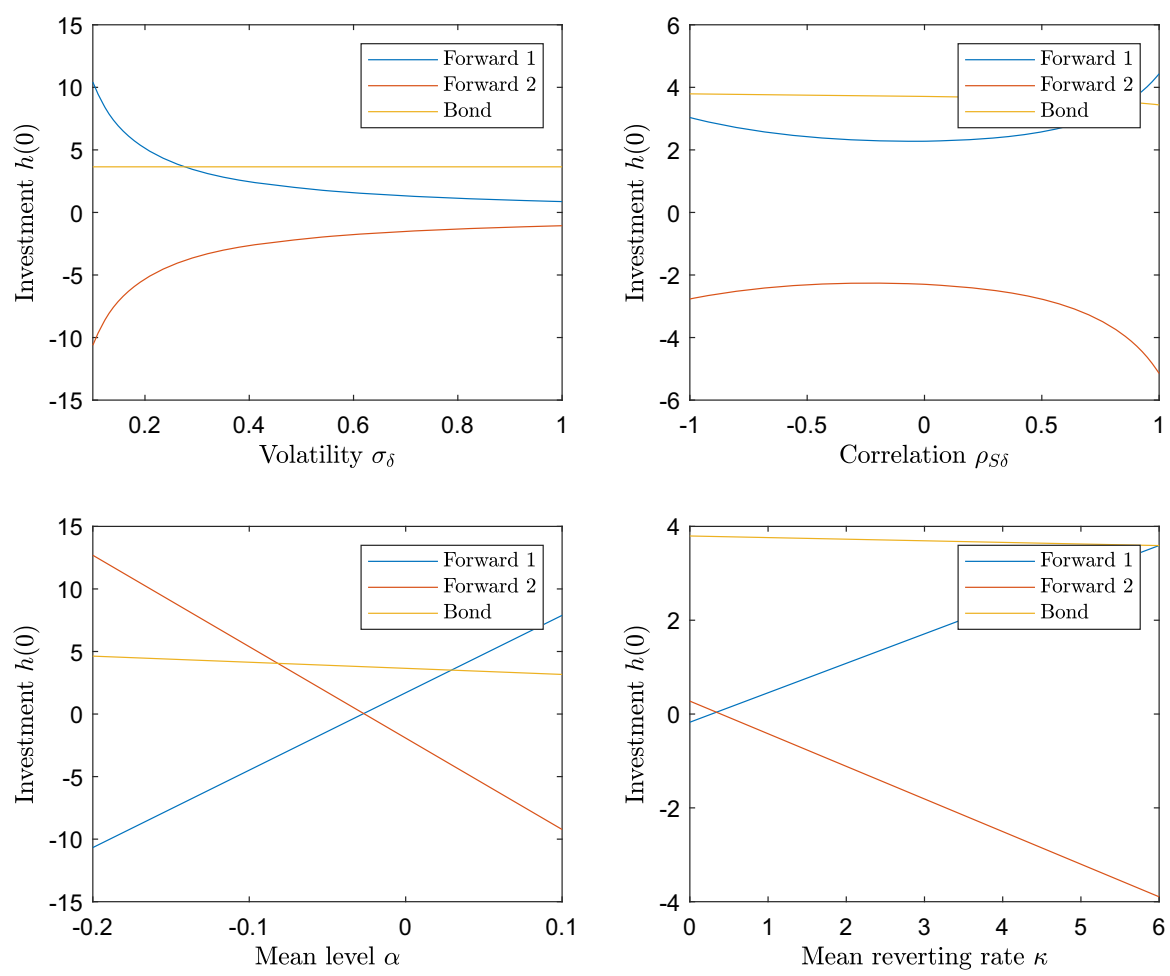

Fig. 1 Fractional investments in assets

complete market solution). This is a very common and costly mistake, documented in many papers in the area. Given the lack of analysis on CY, we focus on incompleteness due to failing to hedge CY movements.

\subsubsection{Impact of parameters on optimal strategy}

Figure 1 illustrates the relationship between the optimal allocation versus one of the four parameters associated with the CY: $\sigma_{\delta}, \rho_{S \delta}, \alpha$ and $\kappa$, while keeping all other parameters constant.

These parameters have little impact on the size of the investment in bonds, although there is a slight decrease in the allocation to bond in terms of $\rho_{S \delta}, \alpha$ and $\kappa$. This is likely explained by the low positive correlation between IRs and CY (i.e. negative correlation between the return on the bond and CY).

In contrast, all of the parameters significantly impact investments in the two prepaid forwards. The investments in the prepaid forwards looks symmetrical, which means that, on the one hand, the absolute allocation in these risky assets remain constant for changes in these parameters. On the other hand, it highlights a so-called "mis-match investment of maturity". The reason for this comes from Eq. 12, i.e. a shorter maturity implies a larger excess return on the commodity and smaller variance (if $\rho_{S \delta} \geq 0$ ) 
Loss on Mis-specified Volatility and Correlations
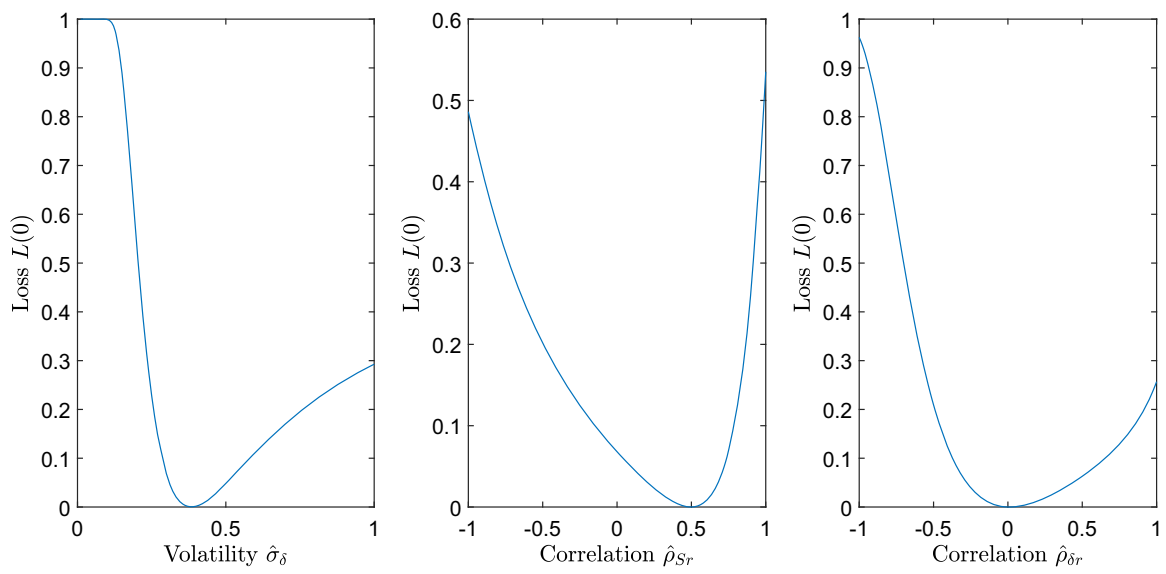

Fig. 2 Loss versus volatility of $\mathrm{CY}$

hence more profitability. Moreover, a larger maturity could lead to prepaid forward negatively correlated to shorter maturities, thus explaining the shift from negative to positive positions.

A larger volatility $\sigma_{\delta}$, and a small mean-reverting rate $\kappa$ tend to decrease the scale of the mis-match investment. The decreasing trend with regard to $\sigma_{\delta}$ is due to the products becoming less appealing to risk-averse investors. Smaller mean-reverting rate $\kappa$ results in a smaller market price of risk $A_{T-t} \lambda_{\delta}$ and volatility $A_{T-t} \sigma_{\delta}$, this can also be seen from Eq. 12. As a result, the increase in overall volatility impacts more than the increase in the drift. Therefore, additional investment is allocated to the shorter-maturity Forward 1.

\subsubsection{Parametric misspecification of convenience yield}

Figure 2 displays WELs with regard to a mis-specified volatility of the CY, as well as misspecifications of the correlations between CY and the other two factors (spot price and IR).

All three plots exhibit relatively large losses due to discrepancies in the real value of the parameters. It must be noted that these are parameters that are not controlled by the robust approach in Maenhout (2004), as these are covariance related and therefore they can not be perturbed via a Girsanov change of measure.

The left plot demonstrates that working with smaller-than-true CY volatilities could be more damaging than working with larger-than-true volatilities. For instance a smaller mis-specified volatility, $\hat{\sigma}_{\delta}$, of approximately 0.15 , when the true volatility is 0.38 , could result in close to $99.5 \%$ WEL. This means that a 5-cent investment by an optimal investor can produce the same utility as a 1-dollar investment for the suboptimal manager. On the other hand, using a $\hat{\sigma}_{\delta}$ of about only 1 leads to a $30 \%$ WEL. 

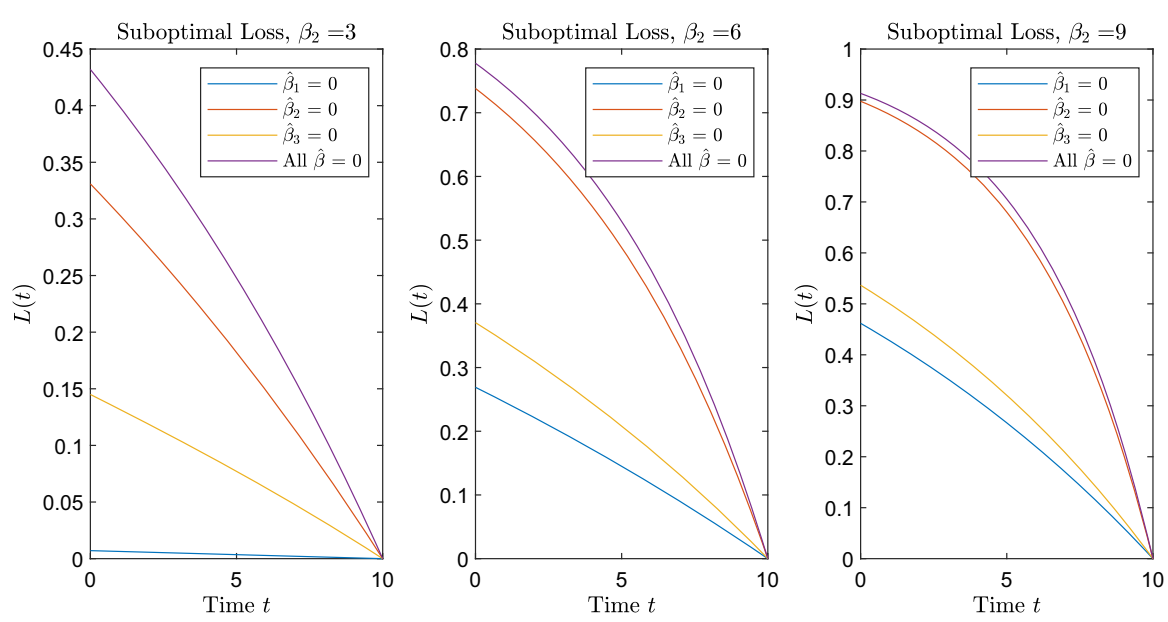

Fig. 3 Wealth-equivalent loss for ignoring ambiguities

For a mis-specified $\hat{\rho}_{S r}$, the perfect correlations ( -1 and 1$)$ lead to roughly a $50 \%$ WEL. In contrast, a mis-specified $\hat{\rho}_{\delta r}$ to -1 and 1 leads to $95 \%$ and $25 \%$ WELs respectively. This indicates that misspecifications of CY correlations play a lesser role in the performance of a commodities portfolio.

\subsubsection{Ignoring ambiguity-aversion}

Similarly to Branger and Larsen (2013) and Escobar et al. (2015), among others, we compute the WEL when an ambiguity averse-investor decides to follow the portfolio allocation that either ignores model uncertainty or works with the wrong level.

For the first subfigure in Fig. 3, we assume that the actual level of ambiguityaversion is $\beta_{i}=3$, for $i=1,2,3$, but the investor acts suboptimally; that is, our computation uses the strategy from 27 while forcing either $\hat{\beta}_{1}=0$ (i.e. ignoring spot price uncertainty), $\hat{\beta}_{2}=0$ (i.e. ignoring $\mathrm{CY}$ uncertainty) or $\hat{\beta}_{3}=0$ (i.e. ignoring IR uncertainty). Each subfigure in Fig. 3 hence displays the WEL for each of the three uncertainties separately, as well as a fourth curve capturing the joint WEL (i.e. the investor ignores all uncertainty $\hat{\beta}_{1}=\hat{\beta}_{2}=\hat{\beta}_{3}=0$ ). The second and third subfigures repeat the analysis assuming $\beta_{2}=6$ and $\beta_{2}=9$ respectively. This means we allow for a higher ambiguity-aversion on CY because it is unobservable, and its parameters can therefore be estimated less accurately.

As can be seen, CY ambiguity version plays the larger role in WEL. In particular, ignoring CY uncertainty can lead to WELs ranging from $34 \%\left(\beta_{2}=3\right)$ to $89 \%$ $\left(\beta_{2}=9\right)$. Furthermore, ignoring IRs uncertainty leads to WELs between $17 \%$ and $52 \%$, while spot uncertainty ranges from $0.01 \%$ to $48 \%$. The low WELs on spot prices are due to the low value of the market price of risk on the commodity $\left(\lambda_{S}=0.1322\right)$, while the simultaneous increase on all WELs from Subfigure 1 Subfigure 3 is due the correlation among the factors. Particularly interesting to see is that ignoring uncertainty 
Suboptimal Loss in Incomplete Markets
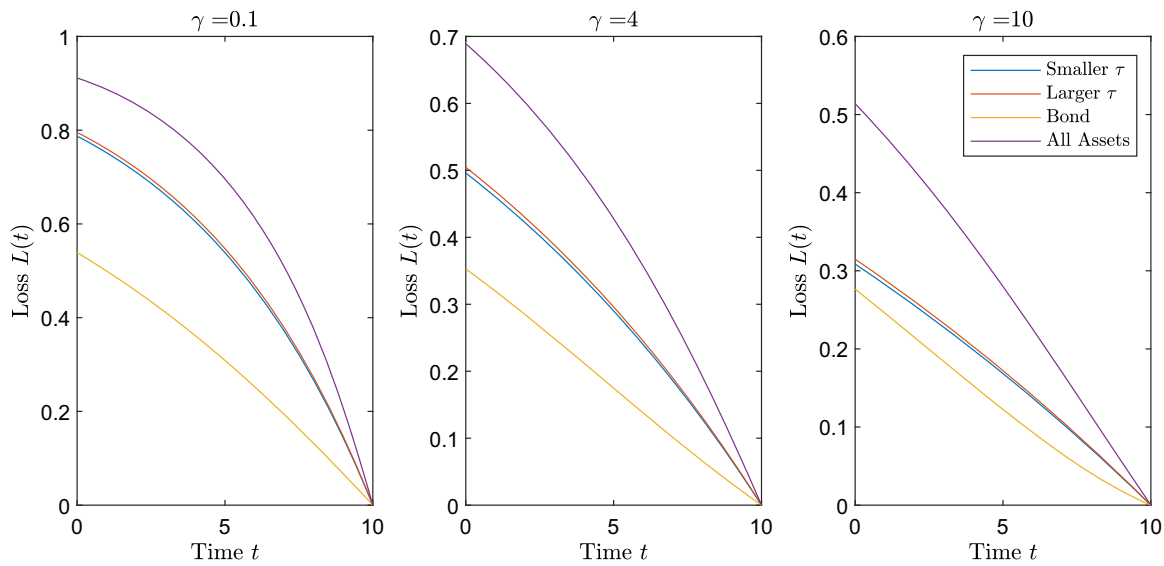

Fig. 4 Wealth-equivalent loss comparison (left: WTI, right: copper)

on all factors together leads to WEL from $43 \%$ to $91 \%$ for the WTI, highlighting the subadditivity of WEL in the context of commodity uncertainty.

\subsubsection{Wealth-equivalent loss due to incomplete market}

It is well known that incomplete markets are a major cause of poor performance in portfolios; see Liu et al. (2005). In this section, we compare the WELs for three types of incomplete markets: absence of a prepaid forward of shorter maturity (e.g. spot price), the absence of longer maturity prepaid forward, and the absence of bonds. We also investigate the extreme case of a cash-only investment.

As indicated in the left of Fig. 4, the smallest WEL comes from the absence of bonds, and it stands at $35 \%$, which is non-negligible. Moreover, incompleteness due to not investing in the spot commodity leads to a larger WEL of approximately $49.5 \%$, while not investing in the longer-maturity prepaid forward yields the largest WEL at $50.5 \%$. Hence, incompleteness due to spot commodity investment is slightly less damaging than avoiding prepaid forwards.

Moreover, the absence of all assets (only investing into a bank account) leads to a WEL of about $69 \%$, confirming again the subadditivity of the individual losses.

\section{Conclusion}

In this paper we studied model uncertainty in the context of portfolio analysis for the asset class of commodities. We focused on a popular model among practitioners (Model III in Schwartz 1997) that highlights the three more important known factors explaining commodity term structures, namely, spot prices, CYs and IRs. We are the first to compare the impact of these three potential sources of uncertainty on the performance of a portfolio. 
In this study we took advantage of the affine structure of the model and the analytical robust portfolio setting of Maenhout (2004) to derive closed-form solutions for an ambiguity-averse utility of terminal wealth maximizer investor. We estimated the parameters of the full model using empirical data on oil future prices and short rates via a combination of MLE and KF.

Our empirical analysis demonstrates that CY is the most influential source of uncertainty for a commodities manager. For WTI, CY is twice as critical as bond prices and substantially more important than spot prices, even under mild assumptions of ambiguity-aversion. For copper, it quickly becomes the most important factor albeit for higher ambiguity-aversion values $\left(\beta_{2}=7\right.$ versus $\left.\beta_{3}=3\right)$. The CY covariancerelated parameters are also critical in terms of damages to the performance of the portfolio due to estimation imprecisions. As a byproduct, we observe that hedging the CY source of risk (i.e. completing the market in terms of CY) is of higher importance compared to the randomness or market-incompleteness from spot prices or IRs.

\section{A Proofs}

\section{A.1 Proof of Proposition 27}

Taking the infimum w.r.t $\boldsymbol{u}$, the change of measure $\boldsymbol{u}$ is

$$
\boldsymbol{u}^{*}=\frac{1}{J(1-\gamma)} \boldsymbol{\beta}_{\rho}\left(J_{x} \boldsymbol{\sigma}^{T} \boldsymbol{\pi}-J_{r} \sigma_{r} \boldsymbol{e}_{3}\right)
$$

Plugging the result lead to the PDE

$$
\begin{aligned}
& \sup _{\boldsymbol{\pi}}\left\{J_{t}+J_{x} r x+\boldsymbol{\pi}^{T} \boldsymbol{\sigma}\left(J_{x} \lambda-J_{x r} \boldsymbol{\rho}_{r} \sigma_{r}+J_{x} J_{r} \frac{\boldsymbol{\beta}_{\rho} \boldsymbol{e}_{3} \sigma_{r}}{J(1-\gamma)}\right)\right. \\
& +a(m-r) J_{r}+\frac{1}{2} J_{r r} \sigma_{r}^{2} \\
& \left.\quad-\frac{J_{r}^{2} \boldsymbol{e}_{3}^{T} \boldsymbol{\beta}_{\rho} \boldsymbol{e}_{3}}{2 J(1-\gamma)} \sigma_{r}^{2}+\frac{1}{2} \boldsymbol{\pi}^{T} \boldsymbol{\sigma}\left(J_{x x} \boldsymbol{\rho}-\frac{J_{x}^{2}}{J(1-\gamma)} \boldsymbol{\beta}_{\rho}\right) \boldsymbol{\sigma}^{T} \boldsymbol{\pi}\right\}=0 .
\end{aligned}
$$

The expression of $\sigma$ indicates a rank of 3. So $\sigma^{T} \pi$ can be projected into the full 3-dimension space. Taking supremum w.r.t $\boldsymbol{\sigma}^{T} \boldsymbol{\pi}$ with a full rank, we obtain

$$
\left(\boldsymbol{\sigma}^{T} \boldsymbol{\pi}\right)^{*}=-\left(J_{x x} \boldsymbol{\rho}-\frac{J_{x}^{2}}{J(1-\gamma)} \boldsymbol{\beta}_{\rho}\right)^{-1}\left(J_{x} \lambda-J_{x r} \boldsymbol{\rho}_{r} \sigma_{r}+J_{x} J_{r} \frac{\boldsymbol{\beta}_{\rho} \boldsymbol{e}_{3} \sigma_{r}}{J(1-\gamma)}\right)
$$

and the final PDE,

$$
J_{t}+J_{x} r x+a(m-r) J_{r}+\frac{\sigma_{r}^{2}}{2}\left(J_{r r}-\frac{J_{r}^{2} \boldsymbol{e}_{3}^{T} \boldsymbol{\beta}_{\rho} \boldsymbol{e}_{3}}{J(1-\gamma)}\right)
$$




$$
\begin{aligned}
& -\frac{1}{2}\left(J_{x} \boldsymbol{\lambda}-J_{x r} \boldsymbol{\rho}_{r} \sigma_{r}+J_{x} J_{r} \frac{\boldsymbol{\beta}_{\rho} \boldsymbol{e}_{3} \sigma_{r}}{J(1-\gamma)}\right)^{T}\left(J_{x x} \rho-\frac{J_{x}^{2}}{J(1-\gamma)} \boldsymbol{\beta}_{\rho}\right)^{-1} \\
& \times\left(J_{x} \boldsymbol{\lambda}-J_{x r} \boldsymbol{\rho}_{r} \sigma_{r}+J_{x} J_{r} \frac{\boldsymbol{\beta}_{\rho} \boldsymbol{e}_{3} \sigma_{r}}{J(1-\gamma)}\right)=0 .
\end{aligned}
$$

Substituting the ansatz $J(t, x, r)=\frac{x^{1-\gamma}}{1-\gamma} \exp \left(A_{0}(t)+A_{1}(t) r\right)$ gives the PDE

$$
\begin{aligned}
A_{0}^{\prime} & +a m A_{1}+\frac{\sigma_{r}^{2}}{2}\left(1-\frac{\boldsymbol{e}_{3}^{T} \boldsymbol{\beta}_{\rho} \boldsymbol{e}_{3}}{1-\gamma}\right) A_{1}^{2} \\
& +\frac{1-\gamma}{2}\left(\lambda+\left(-\boldsymbol{\rho}_{r}+\frac{\boldsymbol{\beta}_{\rho} \boldsymbol{e}_{3}}{1-\gamma}\right) \sigma_{r} A_{1}\right)^{T}\left(\gamma \boldsymbol{\rho}+\boldsymbol{\beta}_{\rho}\right)^{-1} \\
& \times\left(\lambda+\left(-\boldsymbol{\rho}_{r}+\frac{\boldsymbol{\beta}_{\rho} \boldsymbol{e}_{3}}{1-\gamma}\right) \sigma_{r} A_{1}\right)+r\left(A_{1}^{\prime}+1-\gamma-a A_{1}\right)=0 .
\end{aligned}
$$

Utilizing the separation of variables w.r.t. $r$ returns the ODEs with terminal conditions,

$$
\begin{aligned}
& \left\{\begin{array}{l}
A_{0}^{\prime}+a m A_{1}+\frac{\sigma_{r}^{2}}{2}\left(\begin{array}{r}
\left.1-\frac{e_{3}^{T} \beta_{\rho} \boldsymbol{e}_{3}}{J(1-\gamma)}\right) A_{1}^{2}+\frac{1-\gamma}{2}\left(\lambda+\left(-\boldsymbol{\rho}_{r}+\frac{\beta_{\rho} e_{3}}{1-\gamma}\right) \sigma_{r} A_{1}\right)^{T}\left(\gamma \rho+\boldsymbol{\beta}_{\rho}\right)^{-1} \\
\quad \times\left(\lambda+\left(-\boldsymbol{\rho}_{r}+\frac{\beta_{\rho} e_{3}}{1-\gamma}\right) \sigma_{r} A_{1}\right)=0
\end{array}\right. \\
A_{0}(T)=0
\end{array}\right. \\
& \left\{\begin{array}{l}
A_{1}^{\prime}+1-\gamma-a A_{1}=0 \\
A_{1}(T)=0 .
\end{array}\right.
\end{aligned}
$$

Solving the simultaneous ODEs returns the solution in Proposition 27.

\section{A.2 Proof of 1}

First, we present the change of measure $\boldsymbol{u}^{*}$ in the worst-case scenario as a pure function of $t$,

$$
\begin{aligned}
\boldsymbol{u}^{*}(t)= & \frac{1}{J(1-\gamma)} \boldsymbol{\beta}_{\rho}\left(J_{x} \boldsymbol{\sigma}^{T} \boldsymbol{\pi}-J_{r} \sigma_{r} \boldsymbol{e}_{3}\right) \\
= & \frac{\boldsymbol{\beta}_{\boldsymbol{\rho}}}{1-\gamma}\left((1-\gamma)\left(\gamma \rho+\boldsymbol{\beta}_{\rho}\right)^{-1}\left(\lambda+A_{1}(t)\left(-\boldsymbol{\rho}_{r}+\frac{\boldsymbol{\beta}_{\rho} \boldsymbol{e}_{3}}{1-\gamma}\right) \sigma_{r}\right)\right. \\
& \left.-A_{1}(t) \sigma_{r} \boldsymbol{e}_{3}\right)
\end{aligned}
$$

Therefore, given a well-defined set of parameters and the function $\left|A_{1}(t)\right|<\infty$ for $0 \leq t \leq T$, the Novikov's condition for Girsanov's theorem holds, i.e.,

$$
\mathbb{E}^{P}\left[\exp \left(\frac{1}{2} \int_{0}^{T}\left\|\boldsymbol{u}^{*}(t)\right\|\right)\right]<\infty
$$


Hence the change of measure in the worst-case scenario is well-defined. Following Corollary 1.2 in [15] provides the proof that $\pi^{*}$ is in fact the optimal strategy.

\section{A.3 Proof of Proposition 3}

Taking infimum of Eq. 31 w.r.t. $\boldsymbol{u}$, we obtain

$$
\boldsymbol{u}^{*}=\frac{1}{J(1-\gamma)} \boldsymbol{\beta}_{\rho}\left(J_{x} \boldsymbol{\sigma}^{T} \boldsymbol{\pi}_{s}(t)-J_{r} \sigma_{r} \boldsymbol{e}_{3}\right)
$$

The HJB equation is changed into

$$
\begin{gathered}
J_{t}+J_{x} r x+\boldsymbol{\pi}_{s}^{T}(t) \boldsymbol{\sigma}\left(J_{x} \lambda-J_{x r} \boldsymbol{\rho}_{r} \sigma_{r}+J_{x} J_{r} \frac{\boldsymbol{\beta}_{\rho} \boldsymbol{e}_{3} \sigma_{r}}{J(1-\gamma)}\right)+a(m-r) J_{r}+\frac{1}{2} J_{r r} \sigma_{r}^{2} \\
-\frac{J_{r}^{2} \boldsymbol{e}_{3}^{T} \boldsymbol{\beta}_{\rho} \boldsymbol{e}_{3}}{2 J(1-\gamma)} \sigma_{r}^{2}+\frac{1}{2} \boldsymbol{\pi}_{s}^{T}(t) \boldsymbol{\sigma}\left(J_{x x} \boldsymbol{\rho}-\frac{J_{x}^{2}}{J(1-\gamma)} \boldsymbol{\beta}_{\boldsymbol{\rho}}\right) \boldsymbol{\sigma}^{T} \boldsymbol{\pi}_{s}(t)=0 .
\end{gathered}
$$

To simplify the notation, we omit the superscript $s$ of $A_{0}^{s}$ and $A_{1}^{s}$. Plugging the ansatz $J(t, x, r)=\frac{x^{1-\gamma}}{1-\gamma} \exp \left(A_{0}(t)+A_{1}(t) r\right)$ and the assumption $\pi_{s}(t)=x \boldsymbol{h}(t)$ leads to:

$$
\begin{aligned}
A_{0}^{\prime} & +A_{1}^{\prime} r+r(1-\gamma)+(1-\gamma) \boldsymbol{h}^{T}(t) \boldsymbol{\sigma} \lambda-\frac{\gamma(1-\gamma)}{2} \boldsymbol{h}^{T}(t) \boldsymbol{\sigma} \boldsymbol{\rho} \boldsymbol{\sigma}^{T} \boldsymbol{h}(t)+a(m-r) A_{1} \\
& -\frac{1}{2(1-\gamma)}\left((1-\gamma)^{2} \boldsymbol{h}^{T}(t) \boldsymbol{\sigma} \boldsymbol{\beta}_{\rho} \boldsymbol{\sigma}^{T} \boldsymbol{h}(t)\right. \\
& \left.-2(1-\gamma) \boldsymbol{h}^{T}(t) \boldsymbol{\sigma} \boldsymbol{\beta}_{\rho} \boldsymbol{e}_{3} \sigma_{r} A_{1}+\boldsymbol{e}_{3}^{T} \boldsymbol{\beta}_{\rho} \boldsymbol{e}_{3} \sigma_{r}^{2} A_{1}^{2}\right) \\
& +\frac{1}{2} A_{1}^{2} \sigma_{r}^{2}-(1-\gamma) A_{1} \boldsymbol{h}^{T}(t) \boldsymbol{\sigma} \boldsymbol{\rho}_{r} \sigma_{r}=0
\end{aligned}
$$

Via separation of variables w.r.t. $r$, the ODE for the HJB equation is:

$$
\begin{aligned}
& \left\{\begin{array}{l}
A_{1}^{\prime}+1-\gamma-a A_{1}=0 \\
A_{1}(T)=0
\end{array}\right. \\
& \left\{\begin{array}{l}
A_{0}+\left[(1-\gamma) \boldsymbol{h}^{T}(s) \boldsymbol{\sigma} \lambda-\frac{(1-\gamma)}{2} \boldsymbol{h}^{T}(s) \boldsymbol{\sigma}\left(\gamma \boldsymbol{\rho}+\boldsymbol{\beta}_{\boldsymbol{\rho}}\right) \boldsymbol{\sigma}^{T} \boldsymbol{h}(s)+a m A_{1}(s)\right. \\
\left.+\frac{1}{2} A_{1}^{2}(s) \sigma_{r}^{2}\left(1-\frac{\boldsymbol{e}_{3}^{T} \boldsymbol{\beta}_{\rho} \boldsymbol{e}_{3}}{1-\gamma}\right)+A_{1}(s) \boldsymbol{h}^{T}(s) \boldsymbol{\sigma}\left(-(1-\gamma) \boldsymbol{\rho}_{r}+\boldsymbol{\beta}_{\rho} \boldsymbol{e}_{3}\right) \sigma_{r}\right]=0 \\
A_{0}(T)=0 .
\end{array}\right.
\end{aligned}
$$

which returns to the solution in Proposition 3. 


\section{A.4 Proof of Proposition 4}

Taking $\hat{\boldsymbol{\beta}}$ gives us the suboptimal allocation

$$
\boldsymbol{h}(t)=\left[\boldsymbol{\sigma}\left(\gamma \rho+\hat{\boldsymbol{\beta}}_{\rho}\right) \boldsymbol{\sigma}^{T}\right]^{-1} \boldsymbol{\sigma}\left(\lambda+A_{1}(t)\left(-\boldsymbol{\rho}_{r}+\frac{\hat{\boldsymbol{\beta}}_{\rho} \boldsymbol{e}_{3}}{1-\gamma}\right) \sigma_{r}\right)
$$

With the notation $\rho=\boldsymbol{B} \boldsymbol{B}^{T}$ and $\boldsymbol{\beta}_{\rho}=\boldsymbol{B} \boldsymbol{\beta} \boldsymbol{B}^{T}$, plugging the investment allocation into Eq. 32 gives us

$$
\begin{aligned}
A_{0}^{S}(t) & =\int_{t}^{T}-\frac{1-\gamma}{2}\left(\lambda+A_{1}(t)\left(-\boldsymbol{\rho}_{r}+\frac{\hat{\boldsymbol{\beta}}_{\rho} \boldsymbol{e}_{3}}{1-\gamma}\right) \sigma_{r}\right)^{T}\left(\boldsymbol{B}^{T}\right)^{-1}(\gamma \boldsymbol{I}+\hat{\boldsymbol{\beta}})^{-1} \\
& \times(\gamma \boldsymbol{I}+\boldsymbol{\beta})(\gamma \boldsymbol{I}+\hat{\boldsymbol{\beta}})^{-1} \boldsymbol{B}^{-1}\left(\lambda+A_{1}(t)\left(-\hat{\boldsymbol{\rho}}_{r}+\frac{\hat{\boldsymbol{\beta}}_{\rho} \boldsymbol{e}_{3}}{1-\gamma}\right) \sigma_{r}\right) \\
& +\frac{1}{2} A_{1}^{2}(s) \sigma_{r}^{2}\left(1-\frac{\boldsymbol{e}_{3}^{T} \boldsymbol{\beta}_{\rho} \boldsymbol{e}_{3}}{1-\gamma}\right)+a m A_{1}(s) \\
& +(1-\gamma)\left(\lambda+A_{1}(t)\left(-\boldsymbol{\rho}_{r}+\frac{\hat{\boldsymbol{\beta}}_{\rho} \boldsymbol{e}_{3}}{1-\gamma}\right) \sigma_{r}\right)^{T}\left(\boldsymbol{B}^{T}\right)^{-1}(\gamma \boldsymbol{I}+\hat{\boldsymbol{\beta}})^{-1} \\
& \times \boldsymbol{B}^{-1}\left(\lambda+A_{1}(s)\left(-\boldsymbol{\rho}_{r}+\frac{\boldsymbol{\beta}_{\rho} \boldsymbol{e}_{3}}{1-\gamma}\right) \sigma_{r}\right) \mathrm{d} s
\end{aligned}
$$

In order to compute the WEL in Eq. 33, we first compute the difference

$$
\begin{aligned}
A_{0}^{s}(t) & -A_{0}(t) \\
= & \int_{t}^{T}-\frac{1-\gamma}{2}\left(\lambda+A_{1}(s)\left(-\boldsymbol{\rho}_{r}+\frac{\hat{\boldsymbol{\beta}}_{\rho} \boldsymbol{e}_{3}}{1-\gamma}\right) \sigma_{r}\right)^{T}\left(\boldsymbol{B}^{T}\right)^{-1}(\gamma \boldsymbol{I}+\hat{\boldsymbol{\beta}})^{-1} \\
& \times(\gamma \boldsymbol{I}+\boldsymbol{\beta})(\gamma \boldsymbol{I}+\hat{\boldsymbol{\beta}})^{-1} \boldsymbol{B}^{-1}\left(\lambda+A_{1}(s)\left(-\boldsymbol{\rho}_{r}+\frac{\hat{\boldsymbol{\beta}}_{\boldsymbol{\rho}} \boldsymbol{e}_{3}}{1-\gamma}\right) \sigma_{r}\right) \\
& +(1-\gamma)\left(\lambda+A_{1}(s)\left(-\boldsymbol{\rho}_{r}+\frac{\hat{\boldsymbol{\beta}}_{\rho} \boldsymbol{e}_{3}}{1-\gamma}\right) \sigma_{r}\right)^{T}\left(\boldsymbol{B}^{T}\right)^{-1}(\gamma \boldsymbol{I}+\hat{\boldsymbol{\beta}})^{-1} \\
& \times \boldsymbol{B}^{-1}\left(\lambda+A_{1}(s)\left(-\boldsymbol{\rho}_{r}+\frac{\boldsymbol{\beta}_{\rho} \boldsymbol{e}_{3}}{1-\gamma}\right) \sigma_{r}\right) \\
& -\frac{1-\gamma}{2}\left(\lambda+A_{1}(s)\left(-\boldsymbol{\rho}_{r}+\frac{\boldsymbol{\beta}_{\rho} \boldsymbol{e}_{3}}{1-\gamma}\right) \sigma_{r}\right)^{T}\left(\boldsymbol{B}^{-1}\right)^{T}(\gamma \boldsymbol{I}+\boldsymbol{\beta})^{-1} \boldsymbol{B}^{-1} \\
& \times\left(\lambda+A_{1}(s)\left(-\boldsymbol{\rho}_{r}+\frac{\boldsymbol{\beta}_{\rho} \boldsymbol{e}_{3}}{1-\gamma}\right)_{r}\right) \mathrm{d} s .
\end{aligned}
$$


We can easily obtain the quadratic form

$$
\begin{gathered}
A_{0}^{s}(t)-A_{0}(t) \\
=\int_{t}^{T}-\frac{1-\gamma}{2}\left((\gamma \boldsymbol{I}+\boldsymbol{\beta})^{\frac{1}{2}}(\gamma \boldsymbol{I}+\hat{\boldsymbol{\beta}})^{-1} \boldsymbol{B}^{-1}\left(\lambda+A_{1}(s)\left(-\boldsymbol{\rho}_{r}+\frac{\hat{\boldsymbol{\beta}}_{\rho} \boldsymbol{e}_{3}}{1-\gamma}\right) \sigma_{r}\right)\right. \\
\left.-(\gamma \boldsymbol{I}+\boldsymbol{\beta})^{-\frac{1}{2}} \boldsymbol{B}^{-1}\left(\lambda+A_{1}(s)\left(-\boldsymbol{\rho}_{r}+\frac{\boldsymbol{\beta}_{\rho} \boldsymbol{e}_{3}}{1-\gamma}\right) \sigma_{r}\right)\right)^{T} \\
\left((\gamma \boldsymbol{I}+\boldsymbol{\beta})^{\frac{1}{2}}(\gamma \boldsymbol{I}+\hat{\boldsymbol{\beta}})^{-1} \boldsymbol{B}^{-1}\left(\lambda+A_{1}(s)\left(-\boldsymbol{\rho}_{r}+\frac{\hat{\boldsymbol{\beta}}_{\rho} \boldsymbol{e}_{3}}{1-\gamma}\right) \sigma_{r}\right)\right. \\
\left.-(\gamma \boldsymbol{I}+\boldsymbol{\beta})^{-\frac{1}{2}} \boldsymbol{B}^{-1}\left(\lambda+A_{1}(s)\left(-\boldsymbol{\rho}_{r}+\frac{\boldsymbol{\beta}_{\rho} \boldsymbol{e}_{3}}{1-\gamma}\right) \sigma_{r}\right)\right) \mathrm{d} s .
\end{gathered}
$$

Rewriting it:

$$
\begin{aligned}
A_{0}^{s}(t)-A_{0}(t)= & \int_{t}^{T}-\frac{1-\gamma}{2}\left(\left((\gamma \boldsymbol{I}+\boldsymbol{\beta})^{\frac{1}{2}}(\gamma \boldsymbol{I}+\hat{\boldsymbol{\beta}})^{-1}-(\gamma \boldsymbol{I}+\boldsymbol{\beta})^{-\frac{1}{2}}\right) \boldsymbol{B}^{-1}\right. \\
& \left.\left(\lambda+A_{1}(s)\left(-\boldsymbol{\rho}_{r}+\frac{\hat{\boldsymbol{\beta}}_{\rho} \boldsymbol{e}_{3}}{1-\gamma}\right) \sigma_{r}\right)\right)^{T} \\
& \left(\left((\gamma \boldsymbol{I}+\boldsymbol{\beta})^{\frac{1}{2}}(\gamma \boldsymbol{I}+\hat{\boldsymbol{\beta}})^{-1}-(\gamma \boldsymbol{I}+\boldsymbol{\beta})^{-\frac{1}{2}}\right) \boldsymbol{B}^{-1}\right. \\
& \left.\left(\lambda+A_{1}(s)\left(-\boldsymbol{\rho}_{r}+\frac{\hat{\boldsymbol{\beta}}_{\rho} \boldsymbol{e}_{3}}{1-\gamma}\right) \sigma_{r}\right)\right) \mathrm{d} s .
\end{aligned}
$$

Therefore, the WEL is an quadratic function of $\lambda$.

\section{A.5 Proof of Corollary 1}

To simplify and generalize the problem, we consider the quadratic maximization problem of

$$
\max _{\boldsymbol{x} \mid \boldsymbol{x}_{2}=0} \frac{1}{2} \boldsymbol{x}^{T} \boldsymbol{A} \boldsymbol{x}+\boldsymbol{b}^{T} \boldsymbol{x}+c
$$

where we set

$$
\boldsymbol{x}=\left[\begin{array}{l}
\boldsymbol{x}_{1} \\
\boldsymbol{x}_{2}
\end{array}\right], \quad \boldsymbol{b}=\left[\begin{array}{l}
\boldsymbol{b}_{1} \\
\boldsymbol{b}_{2}
\end{array}\right], \quad \boldsymbol{A}=\left[\begin{array}{ll}
\boldsymbol{A}_{11} & \boldsymbol{A}_{12} \\
\boldsymbol{A}_{12}^{T} & \boldsymbol{A}_{22}
\end{array}\right]
$$


Table 2 Parameters from calibration

\begin{tabular}{llll}
\hline Parameters & & & \\
\hline Convenience yield & Mean-reverting rate & $\kappa$ & 1.7874 \\
& Risk-neutral measure & $\hat{\alpha}$ & $-2.33 \%$ \\
& Real-world measure & $\alpha$ & $1.60 \%$ \\
& Market price of risk & 0.5122 \\
& Volatility & $\lambda_{\delta}$ & $13.70 \%$ \\
Interest rate & Mean-reverting rate & $\sigma_{\delta}$ & 0.2798 \\
& Real-world measure interest & $a$ & $0.90 \%$ \\
& Market price of risk & $m$ & 0.6803 \\
Commodities & Volatility & $\lambda_{r}$ & $0.98 \%$ \\
& Market price of risk & $\sigma_{r}$ & 0.4270 \\
& Volatility & $\lambda_{S}$ & $27.47 \%$ \\
Correlation & Maturity 1 & $\sigma_{S}$ & 0 \\
& Maturity 2 & $\tau_{1}$ & $1 / 12$ \\
& Commodity \& CY & $\tau_{2}$ & $31.04 \%$ \\
& Commodity \& IR & $\rho_{S \delta}$ & $6.76 \%$ \\
& IR \& CY & $\rho_{S r}$ & $12.58 \%$ \\
\hline
\end{tabular}

Then the quadratic maximizer point would be:

$$
\boldsymbol{x}^{*}=-\boldsymbol{A}_{1}^{-1} \boldsymbol{b}_{1}=-\left[\begin{array}{cc}
\boldsymbol{A}_{11}^{-1} & \mathbf{0} \\
\mathbf{0} & \mathbf{0}
\end{array}\right] \boldsymbol{b}
$$

\section{B Estimation and results with copper future data}

The copper future price data is available from Wiki Continuous Futures ${ }^{5}$ using the code "CME_HG1.". To fit the data structure, we chose weekly future prices with maturity of 1-9 months over the period of Aug 2001-Jan 2020 (the future prices in Nov 2011 are missing so we ignored this period). However, the spot copper price is not available via this website; hence, for simplicity, and adapting the formulas accordingly, we took the 1-month future as the spot price, and $n$-month future to be the $(n-1)$-month future price. Applying MLE and KF with the available dataset leads to the estimations provided in Table 2 below.

We created for copper data figures similar to the WTI analyses in Figs. 3 and 4. In Fig. 5, the plot depicts the WEL due to ignoring ambiguities. The absence of ambiguities on the spot commodity, CY, and IR lead to WEL of 7\%, 6\% and $19.5 \%$ respectively. For a larger ambiguity aversion level on CY (i.e. $\beta_{2}=9$, the last subfigure), the WEL from CY goes up to $31 \%$, larger than the $27 \%$ from ignoring ambiguity on bonds or the $16 \%$ from ignoring ambiguity on spot prices.

\footnotetext{
5 https://www.quandl.com/data/CHRIS-Wiki-Continuous-Futures.
} 

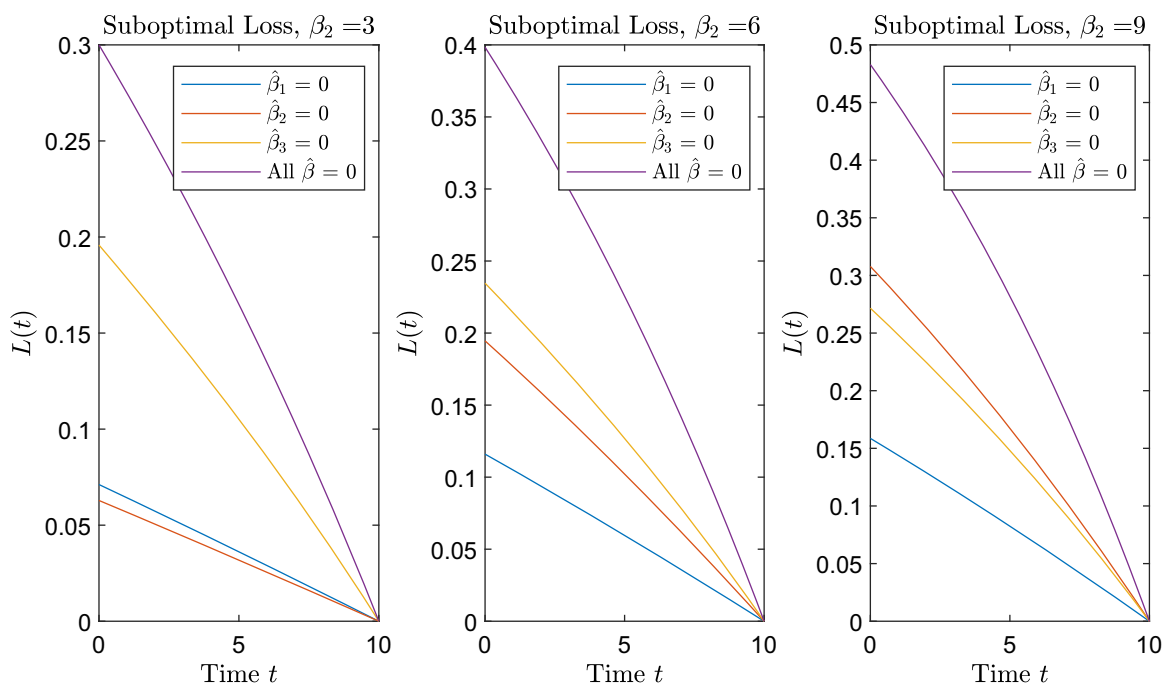

Fig. 5 Wealth-equivalent loss for ignoring ambiguities

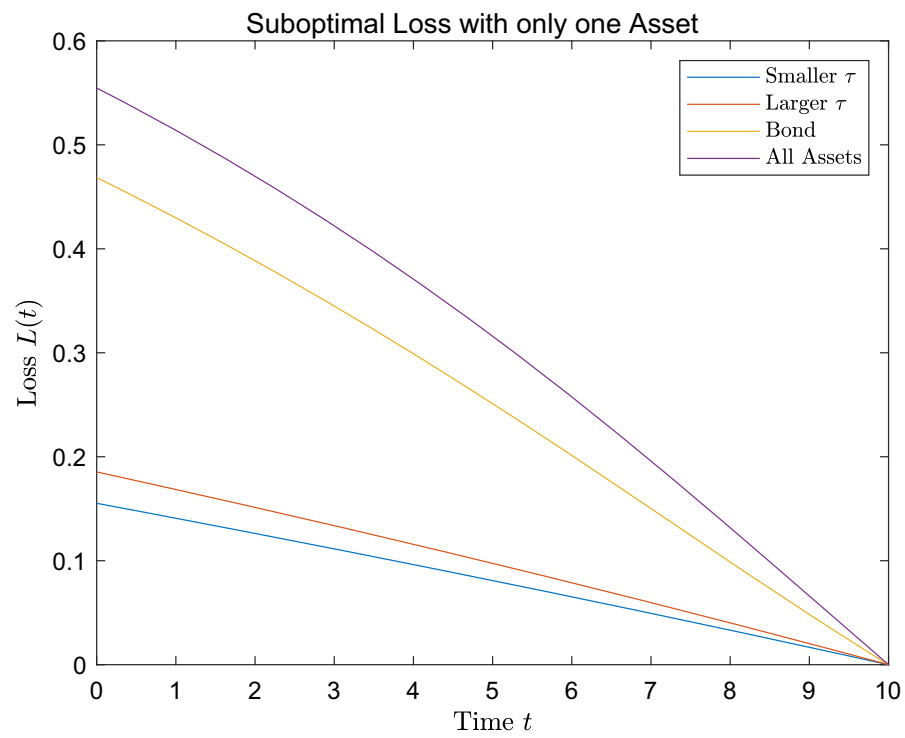

Fig. 6 Wealth-equivalent loss for Incomplete Markets

As for incompleteness of the market, the plot in Fig. 6 illustrates that not investing in bond accounts for $44 \%$ of WELs, which is a larger than WELs due to incompleteness of the spot (15\%) and the longer-maturity prepaid forward (18\%). Moreover, the absence of all assets leads to WEL of 55\%, also satisfying the subadditivity of individual losses. 


\section{References}

Anderson, E., Hansen, L.P., Sargent, T.J.: A quartet of semi-groups for model specification, robustness, prices of risk, and model detection. J Eur Econ Assoc 1(1), 68-123 (2003)

Benth, F.E., Lempa, J.: Optimal portfolios in commodity futures markets. Finance Stochast 18, 407-430 (2014)

Bergen, V., Escobar, M., Rubtsov, A., Zagst, R.: Robust multivariate portfolio choice with stochastic covariance in the presence of ambiguity. Quant Finance 18(8), 1265-1294 (2018)

Branger, N., Larsen, L.: Robust portfolio choice with uncertainty about jump and diffusion risk. J Bank Finance 37(12), 5036-5047 (2013)

Cartea, A., Jaimungal, S., Qin, Z.: Model uncertainty in commodity markets. SIAM J Financial Math 7(1), 1-33 (2016)

Chen, J., Davison, M., Escobar-Anel, M., Zafari, G.: Robust portfolio with commodities and stochastic interest rates. Quant Finance 21, 1-20 (2021)

Chiu, M.C., Wong, H.Y.: Optimal investment for an insurer with cointegrated assets: CRRA utility. Insur Math Econ 52(1), 52-64 (2013)

Cooke, D.A., Gavin, W.T.: Three scenarios for interest rates in the transition to normalcy. Federal Reser Bank St. Louis Rev 97(1), 1-24 (2015)

Ellsberg, D.: Risk, ambiguity, and the savage axioms. Q J Econ 75(4), 643-669 (1961)

Escobar, M., Ferrando, S., Rubtsov, A.: Robust portfolio choice with derivative trading under stochastic volatility. J Bank Finance 61, 142-157 (2015)

Escobar, M., Ferrando, S., Rubtsov, A.: Optimal investment under multi-factor stochastic volatility. Quant Finance 17(2), 241-260 (2016)

Flor, C.R., Larsen, L.S.: Rubost portfolio with stochastic interest rates. Ann Finance 10(2), 243-265 (2013)

Fouque, J.P., Pun, C.S., Wong, H.Y.: Portfolio optimization with ambiguous correlation and stochastic volatilities. SIAM J Control Optim 54(5), 2309-2338 (2016)

Gilboa, I., Schmeidler, D.: Maxmin expected utility with a non-unique prior. J Math Econ 18(2), 141-153 (1989)

Kraft, H.: Optimal portfolios and Hestons stochastic volatility model: an explicit solution for power utility. Quant Finance 5(3), 303-313 (2005)

Kraft, H.: Optimal portfolios with stochastic interest rates and defaultable assets (Vol. 540). New York: Springer

Liu, H.: Dynamic portfolio choice under ambiguity and regime switching mean returns. J Econ Dyn Control 35(4), 623-640 (2011)

Liu, J., Pan, J., Wang, T.: An equilibrium model of rare-event premia and its implication for option smirks. Rev Financial Stud 18(1), 131-164 (2005)

Maenhout, P.J.: Robust portfolio rules and asset pricing. Rev Financial Stud 17(4), 951-983 (2004)

Mataramvura, S., Øksendal, B.: Risk minimizing portfolios and HJBI equations for stochastic differential games. Stochastics 80(4), 317-337 (2008)

Mellios, C., Six, P., Lai, A.N.: Dynamic speculation and hedging in commodity futures markets with a stochastic convenience yield. Eur J Oper Res 250(2), 493-504 (2016)

Merton, R.: Optimum consumption and portfolio rules in a continuous-time model. J Econ Theory 3(4), 373-413 (1971)

Munk, C., Rubtsov, A.: Portfolio choice with stochastic interest rates and inflation ambiguity. Ann Finance 10(3), 419-455 (2014)

Munk, C., Sørensen, C., Vinther, T.N.: Dynamic asset allocation under mean-reverting returns, stochastic interest rates, and inflation uncertainty: are popular recommendations consistent with rational behavior? Int Rev Econ Finance 13(2), 141-166 (2004)

Schwartz, E.S.: The stochastic behavior of commodity prices: implications for valuation and hedging. J Finance 52(3), 923-973 (1997)

Schwartz, E.S., Smith, J.E.: Short-term variations and long-term dynamics in commodity prices. Manage Sci 46(7), 893-911 (2000)

Yan, W., Li, W.: A class of portfolio selection with a four-factor futures price model. Ann Oper Res 164, 139-165 (2008)

Publisher's Note Springer Nature remains neutral with regard to jurisdictional claims in published maps and institutional affiliations. 